\title{
Generating lepton masses and mixings with a heavy vector doublet.
}

\author{
A. E. Cárcamo Hernández ${ }^{*}$ Jonatan Vignatti $\left.\right|^{\dagger}$ and Alfonso Zerwekh \\ Universidad Técnica Federico Santa María \\ and Centro Científico-Tecnológico de Valparaíso \\ Casilla 110-V, Valparaíso, Chile,
}

(Dated: March 5, 2022)

\begin{abstract}
We construct two viable extensions of the SM with a heavy vector in the fundamental $S U(2)_{L}$ representation and nine SM singlet scalar fields, consistent with the current SM fermion mass spectrum and fermionic mixing parameters. The small masses for the active neutrinos are generated from radiative seesaw mechanism at one loop level mediated by the neutral components of the heavy vector as well as by the left handed Majorana neutrinos. The proposed models predicts rates for charged lepton flavor violating processes within the reach of the forthcoming experiments.
\end{abstract}

\section{INTRODUCTION}

In spite of its remarkable agreement with the experimental data, the Standard Model (SM) leaves unexplained several issues such as, for example, the number of fermion families, the observed hierarchical fermion mass spectrum and mixing angles, etc. Whereas the CKM quark mixing matrix is nearly diagonal thus implying small quark mixing angles, the PMNS leptonic mixing matrix significantly deviates from the identity matrix thus giving rise to sizeable leptonic mixing angles. The experimental data indicates that two of the leptonic mixing angles are large whereas one mixing angle is Cabibbo sized, which contrast with the small values of the CKM paramters. In addition, the pattern of fermion masses in the SM is extended over a range of about thirteen orders of magnitude ranging from the light active neutrino mass scale up to the top quark mass. This is so called SM flavor puzzle which is left unaddressed in the SM.

That SM "flavor puzzle" motivates to build models with additional scalars and fermions in their particle spectrum and with an extended gauge group, supplemented by discrete flavour symmetries, which are usually spontaneously broken, in order to generate the observed pattern of SM fermion masses and mixing angles. Recent reviews of discrete flavor groups can be found in Refs. [1 15. Several discrete groups such as $S_{3}$ [6 32, $A_{4}$ 33 74, $S_{4}$ [75, 75 93, $D_{4}$ [94 102, $Q_{6}\left[103-113, T_{7}\right.$ [14-123, $T_{13}$ [124-127, $T^{\prime}$ [128 149], $\Delta(27)$ 150-176], $\Delta(96)$ [177-179], $\Delta\left(6 N^{2}\right)$ [180 182] and $A_{5}$ 183 194 have been implemented in extensions of the SM, to provide a nice description of the observed pattern of fermion masses and mixing angles.

On the other hand, given the current lack of experimental evidence in favor of the traditional big paradigms of Physics beyond the Standard Model, it seems prudent to explore more exotic paths. In recent years, for instance, some groups have pay attention to spin-1 fields transforming in the fundamental representation of $S U(2)_{L}[195$. This kind of field may naturally appear, for instance, in models such as: Higgs-Gauge Unification[196 and Composite Higgs [197, 198. In a previous paper, our group has studied the phenomenology of a spin-1 doublet which has the same hypercharge of the Higgs doublet [199. In the context of Composite Higgs, this kind of field may be interpreted as a spin-1 excitation of the Higgs field in analogy to the rho-mesons which can be seen as the spin-1 excitation of the pions. Such a spin-1 doublet cannot be coupled to standard fermions except by the introduction of exotic fields. The simplest alternative is the introduction of a left-handed fermion which is singlet under the SM group. Of course, such an exotic fermion will behave like a sterile neutrino. In this case, the spin- 1 doublet will couple the standard leptons to new exotic neutrino. The aim of this work is to show that the introduction of such new fields (the spin-1 doublet and the new sterile left-handed neutrino) can have an impact on neutrino Physics by providing a new mechanism for the mass generation of the light active neutrinos.

In this paper, we extend the Standard Model by introducing a new vector doublet field. We treat it as matter field and the resulting model is thought as an effective theory. In other words, we will not discuss the details of the dynamics that generates this vector doublet field and its mass which is beyond the scope of this article. The consistency of such a construction with perturbative unitarity and collider phenomenology have been studied elsewhere [199.

\footnotetext{
*Electronic address: antonio.carcamo@usm.cl

${ }^{\dagger}$ Electronic address: jonatan.vignatti@sansano.usm.cl

$\ddagger$ Electronic address: alfonso.zerwekh@usm.cl
} 
Additionally, we supplement SM gauge symmetry by including a $S_{3}$ discrete group as well as several cyclic symmetries and we extend the particle content by adding several gauge singlet scalars, two left handed neutrinos $N_{n L}(n=1,2)$, singlets under the SM gauge group and a $S U(2)_{L}$ doublet of heavy vectors. We introduce several gauge singlet scalar fields to provide an explanation for the observed hierarchies in the SM fermion mass spectrum and the fermion mixing parameters while keeping all the Yukawa couplings of order unity. We consider two models: The first one is valid only for the inverted neutrino mass hierarchy whereas the second one allows to successfully account for lepton masses and mixing in the scenario of normal neutrino mass ordering. These models are consistent with the SM fermion masses and mixings. The effective neutrino mass matrix arises through radiative seesaw.

The paper is organized as follows. In section II we explain the first model. In Sec. III we focus on the discussion of quark masses and mixing and give our corresponding results. In Sec. IV] we discuss the implications of our model on lepton masses and mixings. In section $\mathrm{V}$ we propose an alternative model in order to account for lepton masses and mixings for the case of normal neutrino mass hierarchy. Section VI provides a discussion of the charged lepton flavor violation constraints on the model parameter space. We conclude in section VII Appendix A provides a concise description of the $S_{3}$ discrete group.

\section{THE MODEL}

We propose an extension of the Standard Model (SM) where the SM gauge symmetry is supplemented by the $S_{3} \times Z_{2} \times$ $Z_{6} \times Z_{8} \times Z_{12}$ discrete group and the particle content is extended to include the SM scalars singlets $\varphi, \chi, \xi, \eta, \sigma, \rho_{k}(k=$ $1,2)$, two left handed neutrinos $N_{n L}(n=1,2)$, singlets under the SM gauge group and a $S U(2)_{L}$ doublet of heavy vectors. The full symmetry $\mathcal{G}$ of our model experiences the following two step spontaneous breaking:

$$
\begin{gathered}
\mathcal{G}=S U(3)_{C} \times S U(2)_{L} \times U(1)_{Y} \times S_{3} \times Z_{2} \times Z_{6} \times Z_{8} \times Z_{12} \\
\Downarrow \Lambda_{\text {int }} \\
S U(3)_{C} \times S U(2)_{L} \times U(1)_{Y} \\
\Downarrow v \\
S U(3)_{C} \times U(1)_{Q}
\end{gathered}
$$

where the symmetry breaking scales satisfy the hierarchy $\Lambda_{i n t}>v$ and $v=246 \mathrm{GeV}$ is the electroweak symmetry breaking scale.

The vector doublet is described by the following Lagrangian:

$$
\begin{aligned}
\mathcal{L} & =-\frac{1}{2}\left(D_{\mu} V_{\nu}-D_{\nu} V_{\mu}\right)^{\dagger}\left(D^{\mu} V^{\nu}-D^{\nu} V^{\mu}\right)+M_{V}^{2} V_{\mu}^{\dagger} V^{\mu} \\
& +\lambda_{2}\left(\phi^{\dagger} \phi\right)\left(V_{\mu}^{\dagger} V^{\mu}\right)+\lambda_{3}\left(\phi^{\dagger} V_{\mu}\right)\left(V^{\mu \dagger} \phi\right)+\lambda_{4}\left(\phi^{\dagger} V_{\mu}\right)\left(\phi^{\dagger} V^{\mu}\right) \\
& +\alpha_{1} \phi^{\dagger} D_{\mu} V^{\mu}+\alpha_{2}\left(V_{\mu}^{\dagger} V^{\mu}\right)\left(V_{\nu}^{\dagger} V^{\nu}\right)+\alpha_{3}\left(V_{\mu}^{\dagger} V^{\nu}\right)\left(V_{\nu}^{\dagger} V^{\mu}\right) \\
& +i g \kappa_{1} V_{\mu}^{\dagger} W^{\mu \nu} V_{\nu}+i \frac{g^{\prime}}{2} \kappa_{2} V_{\mu}^{\dagger} B^{\mu \nu} V_{\nu}+\text { h.c. }
\end{aligned}
$$

where $\phi$ is the standard Higgs doublet and $V_{\mu}$ is the new vector doublet.

Notice that the dimension-3 term $\alpha_{1} \phi^{\dagger} D_{\mu} V^{\mu}$ can be safely dropped out (or, equivalently, we can set $\alpha_{1}=0$ ) because in that case an accidental $Z_{2}$ symmetry opens up. In this way, the mixing of the new vectors and the gauge boson is forbidden at tree level. The consequences of this Lagrangian (with $\alpha_{1}=0$ ) in absence of any other New Physics was already studied in detail in Ref. [199]. In this work, we will explore the implications of these new vectors in the generation of masses for light active neutrinos.

In our model the fermion sector is augmented by adding two heavy gauge singlet left handed Majorana neutrinos. The transformation properties of the quark, lepton and scalar fields under the $S_{3} \times Z_{2} \times Z_{6} \times Z_{8} \times Z_{12}$ discrete group are indicated in Tables II II and III, respectively.

Here the dimensions of the $S_{3}$ irreducible representations are specified by the numbers in boldface and the different $Z_{2} \times Z_{6} \times Z_{8} \times Z_{12}$ charges are written in additive notation.

The $S_{3} \times Z_{2} \times Z_{6} \times Z_{8} \times Z_{12}$ assignment of the heavy vector in the fundamental $S U(2)_{L}$ representation is:

$$
V_{\mu} \sim(\mathbf{1}, 1,0,0,0) .
$$




\begin{tabular}{|c|c|c|c|c|c|c|c|c|c|}
\hline & $q_{1 L}$ & $q_{2 L}$ & $q_{3 L}$ & $u_{1 R}$ & $u_{2 R}$ & $u_{3 R}$ & $d_{1 R}$ & $d_{2 R}$ & $d_{3 R}$ \\
\hline$S_{3}$ & $\mathbf{1}$ & $\mathbf{1}$ & $\mathbf{1}$ & $\mathbf{1}$ & $\mathbf{1}$ & $\mathbf{1}$ & $\mathbf{1}^{\prime}$ & $\mathbf{1}^{\prime}$ & $\mathbf{1}^{\prime}$ \\
\hline$Z_{2}$ & 0 & 0 & 0 & 0 & 0 & 0 & 0 & 0 & 0 \\
\hline$Z_{6}$ & 0 & 0 & 0 & 0 & 0 & 0 & 3 & 3 & 3 \\
\hline$Z_{8}$ & -2 & -1 & 0 & 2 & 1 & 0 & 2 & 1 & 0 \\
\hline$Z_{12}$ & 0 & 0 & 0 & 6 & 6 & 0 & 0 & 0 & 0 \\
\hline
\end{tabular}

Table I: Transformation properties of the quark fields under the $S_{3} \times Z_{2} \times Z_{6} \times Z_{8} \times Z_{12}$ discrete group.

\begin{tabular}{|c|c|c|c|c|c|c|c|}
\hline & $l_{1 L}$ & $l_{L}$ & $l_{1 R}$ & $l_{2 R}$ & $l_{3 R}$ & $N_{1 L}$ & $N_{2 L}$ \\
\hline$S_{3}$ & $\mathbf{1}^{\prime}$ & $\mathbf{2}$ & $\mathbf{1}$ & $\mathbf{1}$ & $\mathbf{1}^{\prime}$ & $\mathbf{1}$ & $\mathbf{1}$ \\
\hline$Z_{2}$ & 0 & 0 & 0 & 0 & 0 & 1 & 1 \\
\hline$Z_{6}$ & -2 & -2 & -2 & -2 & -2 & 3 & 3 \\
\hline$Z_{8}$ & -3 & -1 & -1 & 3 & -1 & 4 & 4 \\
\hline$Z_{12}$ & 0 & 0 & 0 & 0 & 6 & 6 & 6 \\
\hline
\end{tabular}

Table II: Transformation properties of the leptonic fields under the $S_{3} \times Z_{2} \times Z_{6} \times Z_{8} \times Z_{12}$ discrete group.

From the point of view of the gauge symmetry, $V_{\mu}$ is assumed to transform as a matter field in the fundamental representation of $S U(2)_{L}$, i.e. $V_{\mu} \rightarrow U V_{\mu}$ with $U \in S U(2)_{L}$. Additionally, we assume that the hypercharge of the new vector double is the same as the SM Higgs doublet. As a consequence, as we will see below, our model results to be similar to the well known Two Higgs Doublet Model with the second scalar doublet replaced by a vector one.

With the particle spectrum previously specified, we get the following quark and lepton Yukawa terms as well as the interaction terms of the heavy vector with the heavy left handed Majorana neutrinos:

$$
\begin{gathered}
\mathcal{L}_{Y}^{(q)}=y_{33}^{(u)} \bar{q}_{3 L} \widetilde{\phi} u_{3 R}+y_{23}^{(u)} \bar{q}_{2 L} \widetilde{\phi} u_{3 R} \frac{\eta}{\Lambda}+y_{13}^{(u)} \bar{q}_{1 L} \widetilde{\phi} u_{3 R} \frac{\eta^{2}}{\Lambda^{2}} \\
+y_{32}^{(u)} \bar{q}_{3 L} \widetilde{\phi} u_{2 R} \frac{\eta \rho_{1}^{2}}{\Lambda^{3}}+y_{22}^{(u)} \bar{q}_{2 L} \widetilde{\phi} u_{2 R} \frac{\eta^{2} \rho_{1}^{2}}{\Lambda^{4}}+y_{12}^{(u)} \bar{q}_{1 L} \widetilde{\phi} u_{2 R} \frac{\eta^{3} \rho_{1}^{2}}{\Lambda^{5}} \\
+y_{11}^{(u)} \bar{q}_{1 L} \widetilde{\phi} u_{1 R} \frac{\eta^{2} \rho_{1}^{2}}{\Lambda^{4}}+y_{21}^{(u)} \bar{q}_{2 L} \widetilde{\phi} u_{1 R} \frac{\eta^{3} \rho_{1}^{2}}{\Lambda^{5}}+y_{11}^{(u)} \bar{q}_{1 L} \widetilde{\phi} u_{1 R} \frac{\eta^{4} \rho_{1}^{2}}{\Lambda^{6}} \\
+\sum_{j=1}^{3} \sum_{k=1}^{3} y_{j k}^{(d)} \bar{q}_{j L} \phi d_{k R} \frac{\eta^{6-j-k}(\xi \xi \xi)_{1^{\prime}}}{\Lambda^{9-j-k}}+h . c \\
\mathcal{L}_{Y}^{(l)}=y_{11}^{(l)} \bar{l}_{1 L} \phi l_{1 R} \frac{\eta^{2} \rho_{2}^{3} \varphi^{4}}{\Lambda^{9}}+y_{22}^{(l)}\left(\bar{l}_{L} \phi \chi\right)_{1} l_{2 R} \frac{\eta^{4}}{\Lambda^{5}}+y_{13}^{(l)} \bar{l}_{1 L} \phi l_{3 R} \frac{\eta^{2} \rho_{1}^{2}}{\Lambda^{4}}+y_{23}^{(l)}\left(\bar{l}_{L} \phi \chi\right)_{1} l_{3 R} \frac{\rho_{2}^{3}}{\Lambda^{4}} \\
+y_{33}^{(l)}\left(\bar{l}_{L} \phi \chi\right)_{1^{\prime}} l_{3 R} \frac{\rho_{1}^{2}}{\Lambda^{3}}+\frac{1}{2} \sum_{n=1}^{2} m_{N_{n}} \bar{N}_{n L} N_{n L}^{C}+h . c \\
-\mathcal{L}_{V l N}=\sum_{n=1}^{2} y_{1 n}^{(V)} \bar{l}_{1 L} \gamma^{\mu} V_{\mu} N_{n L} \frac{\sigma \eta^{4} \rho_{1}^{2} \varphi}{\Lambda^{8}}+\sum_{n=1}^{2} y_{2 n}^{(V)} \bar{l}_{L} \gamma^{\mu} V_{\mu} N_{n L} \frac{\xi \eta^{4} \rho_{1}^{2} \varphi}{\Lambda^{8}}
\end{gathered}
$$

\begin{tabular}{|c|c|c|c|c|c|c|c|c|}
\hline & $\phi$ & $\varphi$ & $\chi$ & $\xi$ & $\eta$ & $\sigma$ & $\rho_{1}$ & $\rho_{2}$ \\
\hline$S_{3}$ & $\mathbf{1}$ & $\mathbf{1}$ & $\mathbf{2}$ & $\mathbf{2}$ & $\mathbf{1}$ & $\mathbf{1}$ & $\mathbf{1}$ & $\mathbf{1}^{\prime}$ \\
\hline$Z_{2}$ & 0 & 0 & 0 & 0 & 0 & 0 & 0 & 0 \\
\hline$Z_{6}$ & 0 & -2 & 0 & -3 & 0 & -3 & 0 & 0 \\
\hline$Z_{8}$ & 0 & -1 & 0 & 0 & -1 & -2 & 0 & 0 \\
\hline$Z_{12}$ & 0 & 0 & 0 & 0 & 0 & 0 & -3 & -2 \\
\hline
\end{tabular}

Table III: Transformation properties of the scalar fields under the $S_{3} \times Z_{2} \times Z_{6} \times Z_{8} \times Z_{12}$ discrete groups. 
where $y_{i j}^{(u, d)}(i, j=1,2,3), y_{11}^{(l)}, y_{22}^{(l)}, y_{13}^{(l)}, y_{23}^{(l)}, y_{33}^{(l)}, y_{1 n}^{(V)}$ and $y_{2 n}^{(V)}$ are dimensionless quantities. Notice that, given the quantum number assignments on the discrete group factors and the transformation properties of the fields under the gauge symmetry, the coupling written in eq. (6) are the only ones allowed involving the new vector doublet and fermions (excepting for higher dimensional operators).

From the interactions terms of the heavy vector with the heavy left handed Majorana neutrinos we find:

$$
-\mathcal{L}_{V l N} \supset \sum_{n=1}^{2} y_{1 n}^{(V)} \bar{l}_{1 L} \gamma^{\mu} V_{\mu} N_{n L} \frac{v_{\sigma} v_{\eta}^{4} v_{\rho_{1}}^{2} v_{\varphi}}{\Lambda^{8}}+\sum_{n=1}^{2} y_{2 n}^{(V)}\left(\bar{l}_{2 L}+\sqrt{2} \bar{l}_{3 L}\right) \gamma^{\mu} V_{\mu} N_{n L} \frac{v_{\xi} v_{\eta}^{4} v_{\rho_{1}}^{2} v_{\varphi}}{\Lambda^{8}}
$$

Besides that, as the hierarchy among charged fermion masses and quark mixing angles mass emerges from the breaking of the $S_{3} \times Z_{6} \times Z_{8} \times Z_{12}$ discrete group, we set the VEVs of the SM singlet scalar fields with respect to the Wolfenstein parameter $\lambda=0.225$, and the model cutoff $\Lambda$, as follows:

$$
v_{\eta} \sim v_{\rho_{1}} \sim v_{\rho_{2}} \sim v_{\varphi} \sim v_{\chi} \sim v_{\xi}=v_{\sigma}=\Lambda_{\text {int }}=\lambda \Lambda .
$$

The role of the different discrete group factors of the model is explained in the following. The $S_{3}, Z_{6}, Z_{8}$ and $Z_{12}$ discrete groups allow to reduce the number of model parameters and set the SM charged lepton mass hierarchy, which is crucial to get viable textures for the lepton sector consistent with the current pattern of lepton masses and mixings, as we will show in Section IV] We use the $S_{3}$ discrete group because since it is the smallest non-Abelian group that has been considerably studied in the literature. The $S_{3}, Z_{6}, Z_{8}$ and $Z_{12}$ symmetries determine the allowed entries of the charged lepton mass matrix. In addition, the $Z_{6}$ symmetry separates the $S_{3}$ scalar doublet $\chi$ participating in the charged lepton Yukawa interactions from the $S_{3}$ doublet $\xi$ that appear in the neutrino Yukawa terms. Furthermore, the $Z_{8}$ and $Z_{12}$ symmetries are crucial for explaining the tau and muon lepton masses and for providing the Cabbibo sized value for the reactor mixing angle $\theta_{13}$ as well as the Cabbibo sized corrections to the atmospheric mixing angle $\theta_{23}$, without tuning the charged lepton Yukawa couplings. The smallness of the electron mass is explained by the $S_{3}, Z_{6}, Z_{8}$ and $Z_{12}$ discrete symmetries. We use the $Z_{12}$ discrete symmetry since it is the smallest cyclic symmetry that glue $\rho_{1}^{2}$ and $\rho_{2}^{3}$ with $l_{3 R}$, considering $l_{3 R}$ charged under this symmetry. The $Z_{2}$ symmetry, under which only the Majorana neutrinos and the heavy vector are charged, is introduced in order to avoid a tree level type I seesaw mechanism for the generation of the light active neutrino masses.

In order to get predictive and viable fermion sector, we assume that the $S_{3}$ doublet SM singlet scalars $\chi$ and $\xi$ have the following VEV configurations:

$$
\langle\chi\rangle=v_{\chi}(1,0), \quad\langle\xi\rangle=v_{\xi}(1, \sqrt{2}) .
$$

The VEV configurations given above correspond to natural solutions of the minimization equations of the scalar potential for the whole region of parameter space as shown in Ref. [25].

\section{QUARK MASSES AND MIXINGS}

From the quark Yukawa terms of Eq. (4), we get that mass matrices for the SM quarks are:

$$
M_{U}=\left(\begin{array}{ccc}
a_{11}^{(u)} \lambda^{6} & a_{12}^{(u)} \lambda^{5} & a_{13}^{(u)} \lambda^{2} \\
a_{21}^{(u)} \lambda^{5} & a_{22}^{(u)} \lambda^{4} & a_{23}^{(u)} \lambda \\
a_{31}^{(u)} \lambda^{4} & a_{32}^{(u)} \lambda^{3} & a_{33}^{(u)}
\end{array}\right) \frac{v}{\sqrt{2}}, \quad \quad M_{D}=\left(\begin{array}{cccc}
a_{11}^{(d)} \lambda^{7} & a_{12}^{(d)} \lambda^{6} & a_{13}^{(d)} \lambda^{5} \\
a_{21}^{(d)} \lambda^{6} & a_{22}^{(d)} \lambda^{5} & a_{23}^{(d)} \lambda^{4} \\
a_{31}^{(d)} \lambda^{5} & a_{32}^{(d)} \lambda^{4} & a_{33}^{(d)} \lambda^{3}
\end{array}\right) \frac{v}{\sqrt{2}}
$$

with $\lambda=0.225$ being one of the Wolfenstein parameters, $v=246 \mathrm{GeV}$ the scale of breaking of the electroweak gauge symmetry and $a_{i j}^{(u, d)}(i, j=1,2,3)$ are $\mathcal{O}(1)$ parameters. Since the charged fermion mass and quark mixing pattern is caused by the spontaneous breaking of the $S_{3} \times Z_{6} \times Z_{8} \times Z_{12}$ discrete group and in order to simplify the analysis, we adopt the following scenario:

$$
\begin{aligned}
& a_{12}^{(u)}=a_{21}^{(u)}, \quad a_{31}^{(u)}=a_{13}^{(u)}, \quad a_{32}^{(u)}=a_{23}^{(u)}, \\
& a_{12}^{(d)}=\left|a_{12}^{(d)}\right| e^{-i \tau_{1}}, \quad a_{21}^{(d)}=\left|a_{12}^{(d)}\right| e^{i \tau_{1}}, \\
& a_{13}^{(d)}=\left|a_{13}^{(d)}\right| e^{-i \tau_{2}}, \quad a_{31}^{(d)}=\left|a_{13}^{(d)}\right| e^{i \tau_{2}}, \quad a_{23}^{(d)}=a_{32}^{(d)} .
\end{aligned}
$$


Furthermore we set $a_{33}^{(u)}=1$, which is suggested by naturalness arguments. Notice that we have chosen the unusual scaling of quark mass textures in Eq. 10 in order to avoid adding more symmetries and scalar fields in our model, that will be required to get several zero entries in the SM quark mass matrices, which are needed to get a more natural scaling in terms of powers of the Wolfenstein parameter.

For the quark mass matrices given above and considering the benchmark scenario previously described, we look for the eigenvalue problem solutions reproducing the experimental values of the quark masses [200, 201, quark mixing parameters and CP violating phase [202], under the condition that the effective parameters $a_{i j}^{(u, d)}(i, j=1,2,3)$ be most close to $\mathcal{O}(1)$. Applying the standard procedure we find the following solution:

$$
\begin{aligned}
& a_{11}^{(u)} \simeq 0.58, \quad a_{22}^{(u)} \simeq 2.19, \quad a_{12}^{(u)} \simeq 0.67, \\
& a_{13}^{(u)} \simeq 0.80, \quad a_{23}^{(u)} \simeq 0.83, \quad a_{11}^{(d)} \simeq 1.96, \\
& a_{12}^{(d)} \simeq 0.53, \quad a_{13}^{(d)} \simeq 1.07, \quad a_{22}^{(d)} \simeq 1.93 \\
& a_{23}^{(d)} \simeq 1.36, \quad a_{33}^{(d)} \simeq 1.35, \quad \tau_{1} \simeq 9.56^{\circ}, \quad \tau_{2} \simeq 4.64^{\circ} .
\end{aligned}
$$

We use the $M_{Z}$-scale experimental values of the quark masses given by Ref. [200] (which are similar to those in

\begin{tabular}{c|l|l}
\hline \hline Observable & Model value & Experimental value \\
\hline$m_{u}(\mathrm{MeV})$ & 1.44 & $1.45_{-0.45}^{+0.56}$ \\
\hline$m_{c}(\mathrm{MeV})$ & 656 & $635 \pm 86$ \\
\hline$m_{t}(\mathrm{GeV})$ & 177.1 & $172.1 \pm 0.6 \pm 0.9$ \\
\hline$m_{d}(\mathrm{MeV})$ & 2.9 & $2.9_{-0.4}^{+0.5}$ \\
\hline$m_{s}(\mathrm{MeV})$ & 57.7 & $57.7_{-15.7}^{+16.8}$ \\
\hline$m_{b}(\mathrm{GeV})$ & 2.82 & $2.82_{-0.04}^{+0.09}$ \\
\hline $\sin \theta_{12}$ & 0.225 & 0.225 \\
\hline $\sin \theta_{23}$ & 0.0412 & 0.0412 \\
\hline $\sin \theta_{13}$ & 0.00351 & 0.00351 \\
\hline$\delta$ & $64^{\circ}$ & $68^{\circ}$ \\
\hline \hline
\end{tabular}

Table IV: Model and experimental values of the quark masses and CKM parameters.

201]). The experimental values of the CKM parameters are taken from Ref. 202. As shown in Table IV] the quark mass spectrum, quark mixing parameters and $\mathrm{CP}$ violating phase obtained in our model are in very good agreement with the experimental data.

\section{LEPTON MASSES AND MIXINGS}

From the interactions terms of the heavy vector with the heavy left handed Majorana neutrinos of Eq. (7), it follows that light active neutrino masses are generated from a one loop level radiative seesaw mechanism mediated by the left handed Majorana neutrinos and by the real and imaginary parts of the neutral components of the heavy vector in the fundamental $S U(2)_{L}$ representation, as illustrated in Fig. 1. Thus, the elements of the light active neutrino 


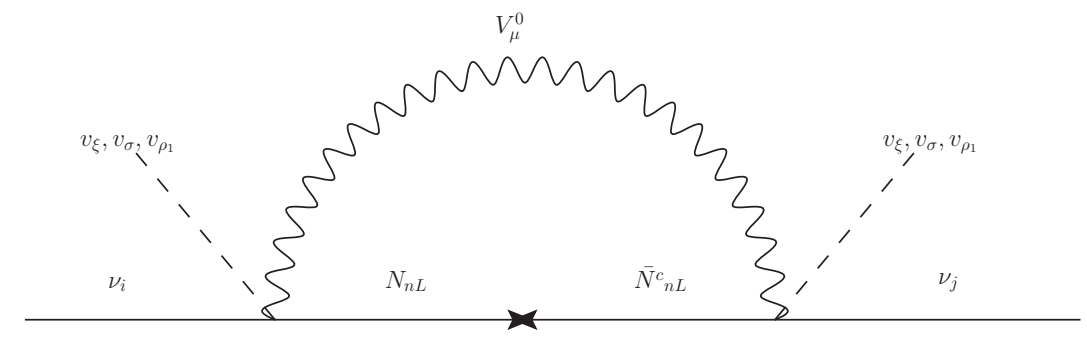

Figure 1: Loop diagram illustrating the generation of the light active neutrino mass. Here $n=1,2$ and $j=1,2,3$.

mass matrix take the form:

$$
\begin{aligned}
& \left(M_{\nu}\right)_{11}=\sum_{n=1}^{2}\left(y_{1 n}^{(V)}\right)^{2} f\left(m_{\operatorname{Re} V^{0}}, m_{\operatorname{Im} V^{0}}, m_{N_{n}}\right)\left(\frac{v_{\xi}}{\Lambda}\right)^{2}\left(\frac{v_{\eta}}{\Lambda}\right)^{8}\left(\frac{v_{\rho_{1}}}{\Lambda}\right)^{4}\left(\frac{v_{\varphi}}{\Lambda}\right)^{2} m_{N_{n}}, \\
& \left(M_{\nu}\right)_{22}=\sum_{n=1}^{2}\left(y_{2 n}^{(V)}\right)^{2} f\left(m_{\operatorname{Re} V^{0}}, m_{\operatorname{Im} V^{0}}, m_{N_{n}}\right)\left(\frac{v_{\xi}}{\Lambda}\right)^{2}\left(\frac{v_{\eta}}{\Lambda}\right)^{8}\left(\frac{v_{\rho_{1}}}{\Lambda}\right)^{4}\left(\frac{v_{\varphi}}{\Lambda}\right)^{2} m_{N_{n}}=2\left(M_{\nu}\right)_{11}, \\
& \left(M_{\nu}\right)_{12}=\left(M_{\nu}\right)_{21}=\sum_{n=1}^{2}\left(y_{1 n}^{(V)}\right)\left(y_{2 n}^{(V)}\right) f\left(m_{\operatorname{Re} V^{0}}, m_{\operatorname{Im} V^{0}}, m_{N_{n}}\right)\left(\frac{v_{\xi}}{\Lambda}\right)^{2}\left(\frac{v_{\eta}}{\Lambda}\right)^{8}\left(\frac{v_{\rho_{1}}}{\Lambda}\right)^{4}\left(\frac{v_{\varphi}}{\Lambda}\right)^{2} m_{N_{n}}=\left(M_{\nu}\right)_{11} \\
& \left(M_{\nu}\right)_{13}=\left(M_{\nu}\right)_{31}=\sqrt{2} \sum_{n=1}^{2}\left(y_{1 n}^{(V)}\right)\left(y_{2 n}^{(V)}\right) f\left(m_{\operatorname{Re} V^{0}}, m_{\operatorname{Im} V^{0}}, m_{N_{n}}\right) \frac{v_{\xi} v_{\sigma}}{\Lambda^{2}}\left(\frac{v_{\eta}}{\Lambda}\right)^{8}\left(\frac{v_{\rho_{1}}}{\Lambda}\right)^{4}\left(\frac{v_{\varphi}}{\Lambda}\right)^{2} m_{N_{n}} \\
& \left(M_{\nu}\right)_{23}=\left(M_{\nu}\right)_{32}=\sqrt{2} \sum_{n=1}^{2}\left(y_{2 n}^{(V)}\right)^{2} f\left(m_{\operatorname{Re} V^{0}}, m_{\operatorname{Im} V^{0}}, m_{N_{n}}\right) \frac{v_{\xi} v_{\sigma}}{\Lambda^{2}}\left(\frac{v_{\eta}}{\Lambda}\right)^{8}\left(\frac{v_{\rho_{1}}}{\Lambda}\right)^{4}\left(\frac{v_{\varphi}}{\Lambda}\right)^{2} m_{N_{n}} \\
& \left(M_{\nu}\right)_{33}=2 \sum_{n=1}^{2}\left(y_{2 n}^{(V)}\right)^{2} f\left(m_{\operatorname{Re} V^{0}}, m_{\operatorname{Im} V^{0}}, m_{N_{n}}\right)\left(\frac{v_{\sigma}}{\Lambda}\right)^{2}\left(\frac{v_{\eta}}{\Lambda}\right)^{8}\left(\frac{v_{\rho_{1}}}{\Lambda}\right)^{4}\left(\frac{v_{\varphi}}{\Lambda}\right)^{2} m_{N_{n}}
\end{aligned}
$$

From the terms given above and the relations given by Eqs. (8) and $(13)$, we find that the charged lepton and light active neutrino mass matrices are respectively given by:

$$
\begin{aligned}
M_{l} & =\frac{v}{\sqrt{2}}\left(\begin{array}{ccc}
a_{1} \lambda^{9} & 0 & a_{4} \lambda^{4} \\
0 & a_{2} \lambda^{5} & a_{5} \lambda^{4} \\
0 & 0 & a_{3} \lambda^{3}
\end{array}\right) \\
M_{\nu} & =\left(\begin{array}{ccc}
Z & Y & \sqrt{2} Y \\
Y & X & \sqrt{2} X \\
\sqrt{2} Y & \sqrt{2} X & 2 X
\end{array}\right) \\
& \simeq \sum_{n=1}^{2}\left(\begin{array}{ccc}
\left(y_{1 n}^{(V)}\right)^{2} & y_{1 n}^{(V)} y_{2 n}^{(V)} & \sqrt{2} y_{1 n}^{(V)} y_{2 n}^{(V)} \\
y_{1 n}^{(V)} y_{2 n}^{(V)} & \left(y_{2 n}^{(V)}\right)^{2} & \sqrt{2}\left(y_{2 n}^{(V)}\right)^{2} \\
\sqrt{2} y_{1 n}^{(V)} y_{2 n}^{(V)} & \sqrt{2}\left(y_{2 n}^{(V)}\right)^{2} & 2\left(y_{2 n}^{(V)}\right)^{2}
\end{array}\right) \lambda^{16} f\left(m_{\left.\operatorname{Re} V^{0}, m_{\operatorname{Im} V^{0}, m_{N_{n}}}\right) m_{N_{n}}}\right.
\end{aligned}
$$




\begin{tabular}{|c||c|c|c|c|}
\hline \multirow{2}{*}{ Observable } & \multirow{2}{*}{ Model value } & \multicolumn{3}{|c|}{ Experimental value } \\
\cline { 3 - 5 } & & $1 \sigma$ range & $2 \sigma$ range & $3 \sigma$ range \\
\hline \hline$m_{e}[\mathrm{MeV}]$ & 0.487 & 0.487 & 0.487 & 0.487 \\
\hline$m_{\mu}[\mathrm{MeV}]$ & 102.8 & $102.8 \pm 0.0003$ & $102.8 \pm 0.0006$ & $102.8 \pm 0.0009$ \\
\hline$m_{\tau}[\mathrm{GeV}]$ & 1.75 & $1.75 \pm 0.0003$ & $1.75 \pm 0.0006$ & $1.75 \pm 0.0009$ \\
\hline$m_{1}[\mathrm{meV}]$ & 49.19 & $\ldots$ & $\ldots$ & $\ldots$ \\
\hline$m_{2}[\mathrm{meV}]$ & 49.96 & $\ldots$ & $\ldots$ & $\ldots$ \\
\hline$m_{3}[\mathrm{meV}]$ & 0 & $\ldots$ & $\ldots$ & $\ldots$ \\
\hline$\Delta m_{21}^{2}\left[10^{-5} \mathrm{eV}^{2}\right](\mathrm{IH})$ & 7.55 & $7.55_{-0.16}^{+0.20}$ & $7.20-7.94$ & $7.05-8.14$ \\
\hline$\Delta m_{13}^{2}\left[10^{-3} \mathrm{eV}^{2}\right](\mathrm{IH})$ & 2.42 & $2.42_{-0.04}^{+0.03}$ & $2.34-2.47$ & $2.31-2.51$ \\
\hline$\delta\left[{ }^{\circ}\right](\mathrm{IH})$ & 309.719 & $281_{-27}^{+23}$ & $229-328$ & $202-349$ \\
\hline $\sin ^{2} \theta_{12} / 10^{-1}(\mathrm{IH})$ & 3.20 & $3.20_{-0.16}^{+0.20}$ & $2.89-3.59$ & $2.73-3.79$ \\
\hline $\sin ^{2} \theta_{23} / 10^{-1}(\mathrm{IH})$ & 5.33 & $5.51_{-0.30}^{+0.18}$ & $4.91-5.84$ & $4.53-5.98$ \\
\hline $\sin ^{2} \theta_{13} / 10^{-2}(\mathrm{IH})$ & 2.248 & $2.220_{-0.076}^{+0.074}$ & $2.07-2.36$ & $1.99-2.44$ \\
\hline
\end{tabular}

Table V: Model and experimental values of the charged lepton masses, neutrino mass squared splittings and leptonic mixing parameters for the inverted (IH) mass hierarchy. The model values for $\mathrm{CP}$ violating phase and the light active neutrino masses are also shown. Notice that we have one massless light active neutrino state since there are two left handed handed Majorana neutrinos mediating the one loop level radiative seesaw mechanism that produces the light active neutrino masses. The experimental values of the charged lepton masses are taken from Ref. [200], whereas the range for experimental values of neutrino mass squared splittings and leptonic mixing parameters, are taken from Ref. [203].

where $a_{1}, a_{2}, b_{1}, c_{1}, y_{1 n}^{(V)}$ and $y_{2 n}^{(V)}$ are $\mathcal{O}(1)$ dimensionless quantities, whereas $X, Y, Z$ are dimensionful parameters which are given by the following relations:

$$
\begin{aligned}
X & \simeq \sum_{n=1}^{2}\left(y_{2 n}^{(V)}\right)^{2} \lambda^{16} f\left(m_{\operatorname{Re} V^{0}}, m_{\operatorname{Im} V^{0}}, m_{N_{n}}\right) m_{N_{n}} \\
Y & \simeq \sum_{n=1}^{2}\left(y_{1 n}^{(V)}\right)\left(y_{2 n}^{(V)}\right) \lambda^{16} f\left(m_{\operatorname{Re} V^{0}}, m_{\operatorname{Im} V^{0}}, m_{N_{n}}\right) m_{N_{n}} \\
Z & \simeq \sum_{n=1}^{2}\left(y_{1 n}^{(V)}\right)^{2} \lambda^{16} f\left(m_{\operatorname{Re} V^{0}}, m_{\operatorname{Im} V^{0}}, m_{N_{n}}\right) m_{N_{n}}
\end{aligned}
$$

being $f\left(m_{\operatorname{Re} V^{0}}, m_{\operatorname{Im} V^{0}}, m_{N_{n}}\right)$ a one loop function:

$$
\begin{aligned}
f\left(m_{\operatorname{Re} V^{0}}, m_{\operatorname{Im} V^{0}}, m_{N_{n}}\right)= & \frac{1}{16 \pi^{2}}\left\{\frac{\Lambda^{2}}{m_{\operatorname{Re} V^{0}}^{2}}-\frac{\Lambda^{2}}{m_{\operatorname{Im} V^{0}}^{2}}+\frac{m_{\operatorname{Re} V^{0}}^{2}}{m_{\operatorname{Re} V^{0}}^{2}-m_{N_{n}}^{2}} \ln \left(\frac{m_{\operatorname{Re} V^{0}}^{2}}{m_{N_{n}}^{2}}\right)-\frac{m_{\operatorname{Im} V^{0}}^{2}}{m_{\operatorname{Im} V^{0}}^{2}-m_{N_{n}}^{2}} \ln \left(\frac{m_{\operatorname{Im} V^{0}}^{2}}{m_{N_{n}}^{2}}\right)\right. \\
& \left.+\left(\frac{m_{N_{n}}^{4}}{m_{\operatorname{Re} V^{0}}^{2}\left(m_{\operatorname{Re} V^{0}}^{2}-m_{N_{n}}^{2}\right)}-\frac{m_{N_{n}}^{2}}{m_{\operatorname{Im} V^{0}}^{2}\left(m_{\operatorname{Im} V^{0}}^{2}-m_{N_{n}}^{2}\right)}\right) \ln \left(\frac{\Lambda^{2}+m_{N_{n}}^{2}}{m_{N_{n}}^{2}}\right)\right\} .
\end{aligned}
$$

Let us note that the loop function has a quadratically divergent part, which arises from the $\frac{q_{\mu} q_{\nu}}{M_{V 0}^{2}}$ term of the heavy vector boson propagator. The charged lepton masses, the neutrino mass squared splittings and the leptonic mixing parameters can be very well reproduced for the scenario of inverted neutrino mass ordering in terms of natural parameters of order one, as shown in Table $\mathrm{V}$, starting from the following benchmark point:

$$
\begin{aligned}
& a_{1} \simeq 1.96168, \quad a_{2} \simeq 1.03698, \quad a_{3} \simeq 0.84294, \quad\left|a_{4}\right| \simeq 1.00752, \quad \arg \left(a_{4}\right) \simeq 218^{\circ}, \\
& a_{5} \simeq-0.597641, \quad X \simeq 16.5289 \mathrm{meV}, \quad Y \simeq-0.219701 \mathrm{meV}, \quad Z \simeq 49.5616 \mathrm{meV},
\end{aligned}
$$

which corresponds to the eigenvalue problem solutions reproducing the experimental values of the neutrino mass squared splittings and leptonic mixing parameters. From the values of the $X, Y$ and $Z$ parameters given above, it is possible to extract, using the light active neutrino mass matrix elements, the mass of the left-handed sterile neutrinos $\left(m_{N}\right)$ for given values of neutrino-heavy-vector coupling and masses for the real and imaginary parts of the neutral components of the heavy vector. As an illustration, we fix the masses of the real and imaginary parts of the neutral 


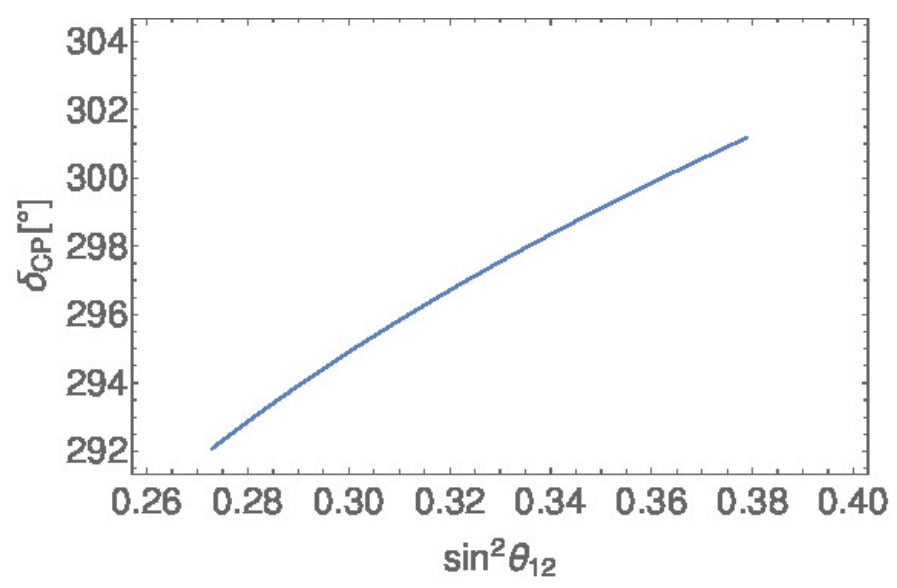

Figure 2: Correlation of the leptonic Dirac CP violating phase $\delta_{C P}$ with the solar $\sin ^{2} \theta_{12}$ mixing parameter.

components of the heavy vector as $m_{\operatorname{Im} V^{0}}=1 \mathrm{TeV}, m_{\mathrm{Re} V^{0}}=0.99 \mathrm{TeV}$ and we consider a neutrino-heavy-vector coupling constant of order unity. For this case we found $m_{N}=245.811 \mathrm{GeV}$. Thus, the heavy vector will decay into an active neutrino and sterile neutrino. Furthermore, the heavy vectors can be pair produced at the LHC via vector boson fusion and Drell-Yan mechanism. In our analysis we have set $\Lambda=3 \mathrm{TeV}$, which is a typical value for the cutoff scale in this kind of models with heavy resonances 204 211. A detailed study of the implications of our model at colliders and dark matter goes beyond the scope of this paper and is deferred for a future work. Studies of Heavy Majorana neutrino pair production at the LHC in the framework of a very general anomaly free $U(1)_{X}$ model have been performed in Refs. 212, 213. In those $U(1)_{X}$ models, a linear seesaw mechanism at tree and one loop level can be implemented to generate the masses for the light active neutrinos and the dark matter relic density can be accommodated by having a scalar dark matter candidate that annihilates into lepton-antilepton pair, where the exchange of charged exotic leptons in the $t$ and $u$ channels mediates this annihilation process, as done in Ref. [214].

In addition, we found a leptonic Dirac CP violating phase of $296.71^{\circ}$ and a Jarlskog invariant close to about $-2.25 \times$ $10^{-2}$ for the inverted hierarchy. Now, let us consider the effective Majorana neutrino mass parameter:

$$
m_{e e}=\left|\sum_{j} U_{e j}^{2} m_{\nu_{j}}\right|,
$$

being $U_{e j}$ the elements of the PMNS matrix and $m_{\nu_{j}}$ the Majorana neutrino masses. The neutrinoless double beta $(0 \nu \beta \beta)$ decay amplitude is proportional to $m_{e e}$. With the model best fit values in Table $\mathrm{V}$ we find

$$
m_{e e} \simeq 46.6813 \mathrm{meV} .
$$

Figures 2 and 3 show the correlations of the leptonic Dirac CP violating phase $\delta_{C P}$ with the solar $\sin ^{2} \theta_{12}$ mixing parameter and the effective Majorana neutrino mass parameter $m_{e e}$ vs the leptonic Dirac CP violating phase $\delta_{C P}$. To obtain these Figures, we randomly varied the lepton sector model parameters around their best fit values in such a way that the resulting neutrino mass squared splittings, leptonic mixing parameters and leptonic Dirac CP violating phase be inside the $3 \sigma$ experimentally allowed range.

As indicated by Figures 2 and 3 , our model predicts solar mixing parameter, leptonic Dirac CP violating phase and effective Majorana neutrino mass parameter in the ranges $0.27 \lesssim \sin ^{2} \theta_{12} \lesssim 0.38,292^{\circ} \lesssim \delta \lesssim 301^{\circ}$ and 0.04655 $\mathrm{eV} \lesssim m_{e e} \lesssim 0.04679 \mathrm{eV}$, respectively.

The range of values given above for the effective Majorana neutrino mass parameter $m_{e e}$ are within the declared reach of the next-generation bolometric CUORE experiment [215] or, more realistically, of the next-to-next-generation tonscale $0 \nu \beta \beta$-decay experiments. The current most stringent experimental upper limit $m_{e e} \leq 160 \mathrm{meV}$ is set by $T_{1 / 2}^{0 \nu \beta \beta}\left({ }^{136} \mathrm{Xe}\right) \geq 1.1 \times 10^{26} \mathrm{yr}$ at $90 \%$ C.L. from the KamLAND-Zen experiment [216]. 


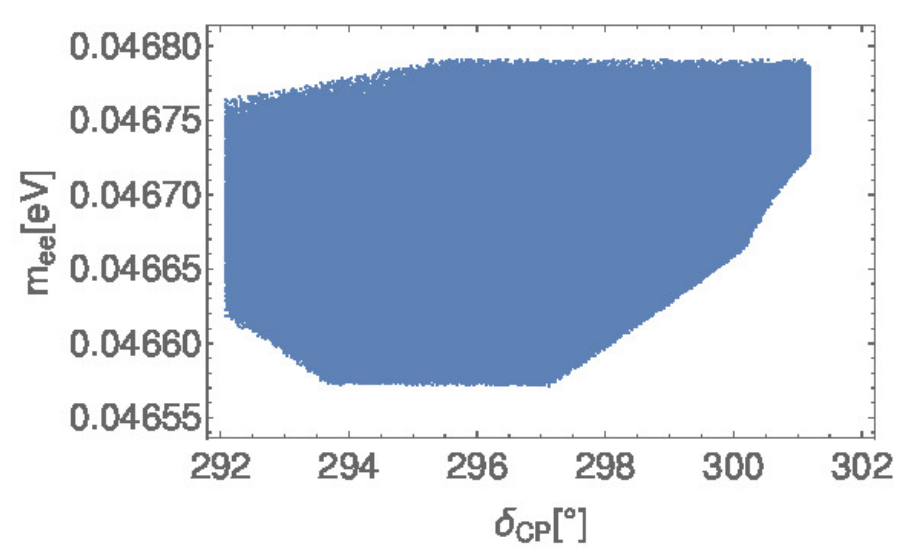

Figure 3: Correlation of the effective Majorana neutrino mass parameter $m_{e e}$ with leptonic Dirac CP violating phase $\delta_{C P}$.

\section{AN ALTERNATIVE MODEL.}

Given that the normal hierarchy is favored over more than $3 \sigma$ than the inverted one, in this section we propose an alternative model based on the $S_{3} \times Z_{3} \times Z_{4} \times Z_{5} \times Z_{8}$ discrete group, where the assignments of the leptonic and scalar fields under such discrete group are shown in Tables VI and VII] respectively. In addition, the $S_{3} \times Z_{3} \times Z_{4} \times Z_{5} \times Z_{8}$ assignment of the heavy vector in the fundamental $S U(2)_{L}$ representation is:

$$
V_{\mu} \sim(\mathbf{1}, 0,2,0,0) .
$$

We consider the following VEV configurations for the $S_{3}$ scalar doublets, which are natural solutions of the scalar potential minimization conditions:

$$
\langle\chi\rangle=v_{\chi}(1,1), \quad\langle\eta\rangle=v_{\chi}(1,-1), \quad\langle\xi\rangle=v_{\xi}(1, \sqrt{2}) .
$$

With the previously specified particle content, we get the following lepton Yukawa terms as well as the interaction terms of the heavy vector with the heavy left handed Majorana neutrinos:

$$
\begin{aligned}
\mathcal{L}_{Y}^{(l)}= & y_{11}^{(l)} \bar{l}_{1 L} \phi l_{1 R} \frac{\varphi \Delta^{4} \sigma^{3} \rho}{\Lambda^{9}}+y_{21}^{(l)} \bar{l}_{L} \phi l_{1 R} \frac{\eta \Delta^{4}}{\Lambda^{9}}\left[\sigma^{3} \rho+x_{1}\left(\sigma^{*}\right)^{2}\left(\rho^{*}\right)^{2}\right] \\
& +y_{12}^{(l)} \bar{l}_{1 L} \phi l_{2 R} \frac{\left(\chi \xi^{*} \xi^{*}\right)_{1^{\prime}} \sigma^{2} \rho}{\Lambda^{6}}+y_{22}^{(l)} \bar{l}_{L} \phi l_{2 R} \frac{\chi}{\Lambda^{9}}\left[\sigma^{3} \rho+x_{2}\left(\sigma^{*}\right)^{2}\left(\rho^{*}\right)^{2}\right] \\
& +y_{13}^{(l)} \bar{l}_{1 L} \phi l_{3 R} \frac{\sigma^{3}}{\Lambda^{3}}+y_{23}^{(l)} \bar{l}_{L} \phi l_{3 R} \frac{\chi \sigma^{3}}{\Lambda^{4}}+y_{33}^{(l)} \bar{l}_{L} \phi l_{3 R} \frac{\chi\left(\sigma^{*}\right)^{2}}{\Lambda^{4}} \\
& +\frac{1}{2} \sum_{n=1}^{2} m_{N_{n}} \bar{N}_{n L} N_{n L}^{C}+h . c \\
-\mathcal{L}_{V l N} & =\sum_{n=1}^{2} y_{1 n}^{(V)} \bar{l}_{1 L} \gamma^{\mu} V_{\mu} N_{n L} \frac{\xi \Delta^{4} \sigma^{2} \rho}{\Lambda^{8}}+\sum_{n=1}^{2} y_{2 n}^{(V)} \bar{l}_{L} \gamma^{\mu} V_{\mu} N_{n L} \frac{\Phi \Delta^{4} \sigma^{2} \rho}{\Lambda^{8}} .
\end{aligned}
$$

From the terms given above and the relations given by Eqs. (8) and (13), we find that the charged lepton and light 


\begin{tabular}{|c|c|c|c|c|c|c|c|}
\hline & $l_{1 L}$ & $l_{L}$ & $l_{1 R}$ & $l_{2 R}$ & $l_{3 R}$ & $N_{1 L}$ & $N_{2 L}$ \\
\hline$S_{3}$ & $\mathbf{1}$ & $\mathbf{2}$ & $1^{\prime}$ & $1^{\prime}$ & $\mathbf{1}^{\prime}$ & $\mathbf{1}$ & $\mathbf{1}$ \\
\hline$Z_{3}$ & -1 & -1 & 0 & 0 & -1 & 0 & 0 \\
\hline$Z_{4}$ & 0 & 0 & 1 & 0 & 0 & 0 & 0 \\
\hline$Z_{5}$ & 1 & 1 & 4 & 4 & 4 & 0 & 0 \\
\hline$Z_{8}$ & 0 & 0 & 4 & 0 & 0 & 4 & 4 \\
\hline
\end{tabular}

Table VI: $S_{3} \times Z_{3} \times Z_{4} \times Z_{5} \times Z_{6}$ assignments of the leptonic fields in the alternative model.

\begin{tabular}{|c|c|c|c|c|c|c|c|c|}
\hline & $\phi$ & $\chi$ & $\eta$ & $\xi$ & $\sigma$ & $\varphi$ & $\rho$ & $\Delta$ \\
\hline$S_{3}$ & $\mathbf{1}$ & $\mathbf{2}$ & $\mathbf{2}$ & 2 & $1^{\prime}$ & $\mathbf{1}$ & $\mathbf{1}$ & 1 \\
\hline$Z_{3}$ & 0 & 0 & 0 & 0 & 0 & 0 & -1 & 0 \\
\hline$Z_{4}$ & 0 & 0 & -1 & -2 & 0 & -1 & 0 & 0 \\
\hline$Z_{5}$ & 0 & 0 & 0 & -2 & -1 & 0 & 0 & 0 \\
\hline$Z_{8}$ & 0 & 0 & 0 & 0 & 0 & 0 & 0 & -1 \\
\hline
\end{tabular}

Table VII: $S_{3} \times Z_{3} \times Z_{4} \times Z_{5} \times Z_{6}$ assignments of the scalar fields.

active neutrino mass matrices are respectively given by:

$$
\begin{aligned}
& M_{l}=\left(\begin{array}{lll}
a_{11} \lambda^{9} & a_{12} \lambda^{6} & a_{13} \lambda^{3} \\
a_{21} \lambda^{9} & a_{22} \lambda^{5} & a_{23} \lambda^{4} \\
a_{31} \lambda^{9} & a_{32} \lambda^{5} & a_{33} \lambda^{3}
\end{array}\right) \frac{v}{\sqrt{2}} \\
& M_{\nu}=\left(\begin{array}{ccc}
Z & Y & \sqrt{2} Y \\
Y & X & \sqrt{2} X \\
\sqrt{2} Y & \sqrt{2} X & 2 X
\end{array}\right) \text {, }
\end{aligned}
$$

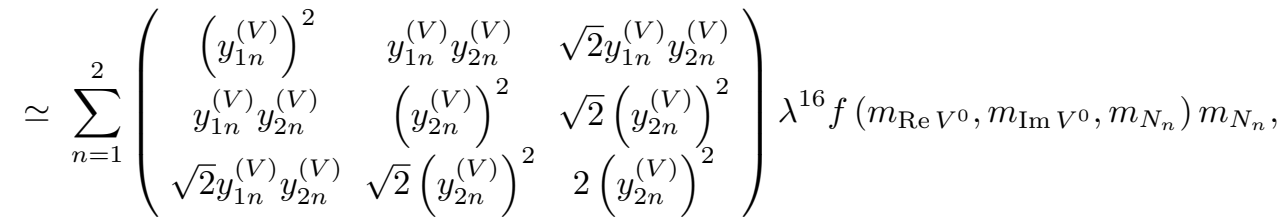

where $a_{11}, a_{12}, a_{13}, a_{21}, a_{22}, a_{23}, a_{31}, a_{32}, a_{33}, y_{1 n}^{(V)}$ and $y_{2 n}^{(V)}$ are $\mathcal{O}(1)$ dimensionless quantities, whereas $X, Y, Z$ are dimensionful parameters which are given by the following relations:

$$
\begin{aligned}
& X \simeq \sum_{n=1}^{2}\left(y_{2 n}^{(V)}\right)^{2} \lambda^{16} f\left(m_{\operatorname{Re} V^{0}}, m_{\operatorname{Im} V^{0}}, m_{N_{n}}\right) m_{N_{n}} \\
& Y \simeq \sum_{n=1}^{2}\left(y_{1 n}^{(V)}\right)\left(y_{2 n}^{(V)}\right) \lambda^{16} f\left(m_{\operatorname{Re} V^{0}}, m_{\operatorname{Im} V^{0}}, m_{N_{n}}\right) m_{N_{n}} \\
& Z \simeq \sum_{n=1}^{2}\left(y_{1 n}^{(V)}\right)^{2} \lambda^{16} f\left(m_{\operatorname{Re} V^{0}}, m_{\operatorname{Im} V^{0}}, m_{N_{n}}\right) m_{N_{n}} .
\end{aligned}
$$

The charged lepton masses, the neutrino mass squared splittings and the leptonic mixing parameters can be very well reproduced for the scenario of normal neutrino mass ordering in terms of natural parameters of order one, as shown in TableVIII starting from the following benchmark point:

$$
\begin{aligned}
& a_{11}=-1.04606+0.07753 i, \quad a_{12}=-0.942761-0.154021 i, \quad a_{13}=0.754455-0.0433344 i, \\
& a_{21}=-1.09641+0.0882596 i, \quad a_{22}=0.807509-0.0359955 i, \quad a_{23}=-0.782795-0.125467 i, \\
& a_{31}=1.1238+0.124818 i, \quad a_{32}=0.59038+0.0469802 i, \quad a_{33}=0.490204+0.0368452 i, \\
& X=13.9813 \mathrm{meV}, \quad Y=9.73655 \mathrm{meV}, \quad Z=17.2485 \mathrm{meV},
\end{aligned}
$$


which corresponds to the eigenvalue problem solutions reproducing the experimental values of the physical observables of the lepton sector: charged lepton masses, neutrino mass squared differences and leptonic mixing parameters. In addition the values of the $X, Y$ and $Z$ parameters given above can be reproduced for the following masses:

$$
m_{\mathrm{Im} V^{0}}=1.5 \mathrm{TeV}, \quad m_{\mathrm{Re} V^{0}}=1.6 \mathrm{TeV}, \quad m_{N}=159 \mathrm{MeV} .
$$

As we will see in the next section, the masses of the neutral components of the heavy vector as well as of the left handed Majorana neutrinos given above, are inside the allowed region of parameter space consistent with the constraints arising for charged lepton flavor violation. In addition, the rates for the charged lepton flavor violating decays obtained for those mass values are within the reach of the forthcoming experiments.

\begin{tabular}{|c|c|c|c|c|}
\hline \multirow{2}{*}{ Observable } & \multirow{2}{*}{ Model Value } & \multicolumn{3}{|c|}{ Experimental value } \\
\cline { 3 - 5 } & & $1 \sigma$ range & $2 \sigma$ range & $3 \sigma$ range \\
\hline \hline$m_{e}[\mathrm{MeV}]$ & 0.487 & 0.487 & 0.487 & 0.487 \\
\hline$m_{\mu}[\mathrm{MeV}]$ & 102.8 & $102.8 \pm 0.0003$ & $102.8 \pm 0.0006$ & $102.8 \pm 0.0009$ \\
\hline$m_{\tau}[\mathrm{GeV}]$ & 1.75 & $1.75 \pm 0.0003$ & $1.75 \pm 0.0006$ & $1.75 \pm 0.0009$ \\
\hline$m_{1}[\mathrm{meV}]$ & 0 & $\ldots$ & $\ldots$ & $\ldots$ \\
\hline$m_{2}[\mathrm{meV}]$ & 8.67 & $\ldots$ & $\ldots$ & $\ldots$ \\
\hline$m_{3}[\mathrm{meV}]$ & 50 & $\ldots$ & $\ldots$ & $\ldots$ \\
\hline$\Delta m_{21}^{2}\left[10^{-5} \mathrm{eV}^{2}\right]$ & 7.55 & $7.55_{-0.16}^{+0.20}$ & $7.20-7.94$ & $7.05-8.14$ \\
\hline$\Delta m_{31}^{2}\left[10^{-3} \mathrm{eV}^{2}\right]$ & 2.50 & $2.50 \pm 0.03$ & $2.44-2.57$ & $2.41-2.60$ \\
\hline $\sin ^{2}\left(\theta_{12}\right) / 10^{-1}$ & 3.20 & $3.20_{-0.16}^{+0.20}$ & $2.89-3.59$ & $2.73-3.79$ \\
\hline $\sin ^{2}\left(\theta_{23}\right) / 10^{-1}$ & 5.47 & $5.47_{-0.30}^{+0.20}$ & $4.67-5.83$ & $4.45-5.99$ \\
\hline $\sin ^{2}\left(\theta_{13}\right) / 10^{-2}$ & 2.160 & $2.160_{-0.069}^{+0.083}$ & $2.03-2.34$ & $1.96-2.41$ \\
\hline$\delta_{C P}$ & $218^{\circ}$ & $218_{-27}^{+38^{\circ}}$ & $182^{\circ}-315^{\circ}$ & $157^{\circ}-349^{\circ}$ \\
\hline
\end{tabular}

Table VIII: Model and experimental values of the charged lepton masses, neutrino mass squared splittings and leptonic mixing parameters for the normal (NH) mass hierarchy. The model values for $\mathrm{CP}$ violating phase and the light active neutrino masses are also shown. Notice that we have one massless light active neutrino state since there are two left handed handed Majorana neutrinos mediating the one loop level radiative seesaw mechanism that produces the light active neutrino masses. The experimental values of the charged lepton masses are taken from Ref. 200, whereas the range for experimental values of neutrino mass squared splittings and leptonic mixing parameters, are taken from Ref. 203.

\section{CHARGED LEPTON FLAVOR VIOLATING DECAY CONSTRAINTS.}

In this section we will determine the constraints that the charged lepton flavor violating (CLFV) decays $\mu \rightarrow e \gamma$, $\tau \rightarrow \mu \gamma$ and $\tau \rightarrow e \gamma$ imposed in the parameter space of our model. These CLFV processes appear at one loop level and are mediated by sterile neutrinos together with the charged components of the heavy vector doublet. The Branching ratio of the $l_{i} \rightarrow l_{j} \gamma$ decay is given by: $[217+219]$ :

$$
\begin{aligned}
B r\left(l_{i} \rightarrow l_{j} \gamma\right) & =\frac{\alpha_{W}^{3} s_{W}^{2} m_{l_{i}}^{5}}{256 \pi^{2} m_{V^{ \pm}}^{4} \Gamma_{i}}\left|\sum_{n=1}^{2} G\left(\frac{m_{N_{n}}^{2}}{m_{V^{ \pm}}^{2}}\right)\right|^{2}, \\
G(x) & =-\frac{2 x^{3}+5 x^{2}-x}{4(1-x)^{2}}-\frac{3 x^{3}}{2(1-x)^{4}} \ln x,
\end{aligned}
$$

where $m_{V^{ \pm}}$are the masses of the charged components of the $S U_{2 L}$ vector doublet $V_{\mu}$, whereas $m_{N_{n}}(n=1,2)$ corresponds to the masses of the left handed Majorana neutrinos $N_{n L}$. To simplify our analysis, we chose a benchmark scenario where the left handed Majorana neutrinos $N_{n L}$ are degenerate with mass equal to $m_{N}$. Figure VI shows the allowed parameter space in the $m_{V^{ \pm}}-m_{N}$ plane consistent with the constraints arising from charged lepton flavor violating decays. As seen from Figure 4 , the obtained values for the branching ratio of $\mu \rightarrow e \gamma$ decay are below its experimental upper limit of $4.2 \times 10^{-13}$ since these values are located in the range $5 \times 10^{-14} \lesssim B r(\mu \rightarrow e \gamma) \lesssim$ $2.5 \times 10^{-13}$, for charged heavy vector and heavy Majorana neutrino masses in the ranges $1.3 \mathrm{TeV} \lesssim m_{V^{ \pm}} \lesssim 2.5 \mathrm{TeV}$ and $100 \mathrm{GeV} \lesssim m_{N} \lesssim 380 \mathrm{GeV}$, respectively. It is worth mentioning that we have considered heavy vectors lighter than about $2.5 \mathrm{TeV}$ in order to comply with the constraints arising from perturbative unitarity [199]. In the same 


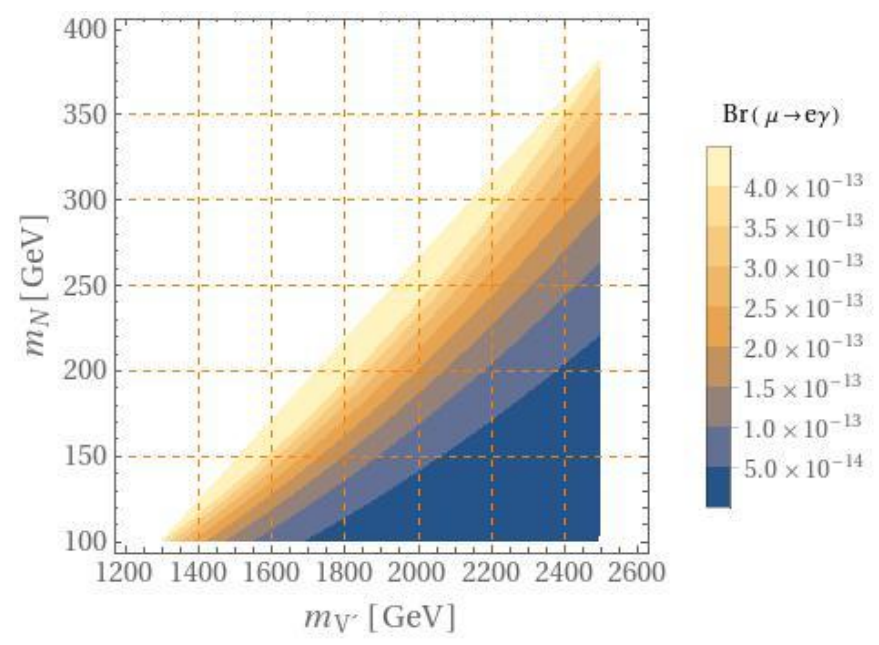

Figure 4: Allowed parameter space in the $m_{V^{ \pm}}-m_{N}$ plane consistent with the LFV constraints.

region of parameter space, the obtained branching ratios for the $\tau \rightarrow \mu \gamma$ and $\tau \rightarrow e \gamma$ decays can reach values of the order of $10^{-10}$, which is below their corresponding upper experimental bounds of $4.4 \times 10^{-8}$ and $3.3 \times 10^{-8}$, respectively. Consequently, our model predicts charged lepton flavor violating processes within the reach of the forthcoming experiments.

\section{CONCLUSIONS}

We have built two viable extensions of the SM with a heavy vector in the fundamental $S U(2)_{L}$ representation and several gauge singlet scalars that successfully explain the observed fermion mass spectrum and mixing parameters at low energies. The models incorporate the $S_{3}$ family symmetry, which is supplemented by other auxiliary cyclic symmetries. The observed hierarchical structure of the SM charged fermion mass spectrum and mixing parameters comes from the spontaneous breaking of both the $S_{3}$ and the different cyclic symmetries. The masses for the light active neutrinos are generated via a one loop level radiative seesaw mechanism. In the first model, the obtained quark mass spectrum, CKM parameters, CP violating phase are compatible with their experimental values, whereas the resulting leptonic mixing parameters agree with current neutrino data only in the case of inverted ordering, featuring in this scenario an excellent agreement. The second model allows to accommodate the experimental values for the physical observables of the lepton sector in the scenario of normal neutrino mass hierarchy. In addition, the proposed models are consistent with the constraints arising from charged lepton flavor violating processes, which restrict the charged heavy vector mass to be larger than about $1.3 \mathrm{TeV}$ and the heavy Majorana neutrino masses in the range $100 \mathrm{GeV} \lesssim m_{N} \lesssim 380 \mathrm{GeV}$. We have found that our model predicts charged lepton flavor violating decays within the reach of the current sensitivity of the forthcoming charged lepton flavor violation experiments

\section{Acknowledgments}

This research has received funding from Fondecyt (Chile), Grants No. 1170803 and No.1160423, CONICYT PIA/Basal FB0821 and PIA/ACT-1406. J.V. acknowledges PIIC/DGIP for supporting this work.

Appendix A: The product rules for $S_{3}$.

The $S_{3}$ discrete group contains 3 irreducible representations: $\mathbf{1}, \mathbf{1}^{\prime}$ and $\mathbf{2}$. Considering $\left(x_{1}, x_{2}\right)^{T}$ and $\left(y_{1}, y_{2}\right)^{T}$ as the basis vectors for two $S_{3}$ doublets and $y^{\prime}$ an $S_{3}$ non trivial singlet, the multiplication rules of the $S_{3}$ group for the case 
of real representations take the form [1]:

$$
\begin{gathered}
\left(\begin{array}{l}
x_{1} \\
x_{2}
\end{array}\right)_{\mathbf{2}} \otimes\left(\begin{array}{l}
y_{1} \\
y_{2}
\end{array}\right)_{\mathbf{2}}=\left(x_{1} y_{1}+x_{2} y_{2}\right)_{\mathbf{1}}+\left(x_{1} y_{2}-x_{2} y_{1}\right)_{\mathbf{1}^{\prime}}+\left(\begin{array}{c}
x_{2} y_{2}-x_{1} y_{1} \\
x_{1} y_{2}+x_{2} y_{1}
\end{array}\right)_{\mathbf{2}} \\
\left(\begin{array}{l}
x_{1} \\
x_{2}
\end{array}\right)_{\mathbf{2}} \otimes\left(y^{\prime}\right)_{\mathbf{1}^{\prime}}=\left(\begin{array}{c}
-x_{2} y^{\prime} \\
x_{1} y^{\prime}
\end{array}\right)_{\mathbf{2}}, \quad\left(x^{\prime}\right)_{\mathbf{1}^{\prime}} \otimes\left(y^{\prime}\right)_{\mathbf{1}^{\prime}}=\left(x^{\prime} y\right)_{\mathbf{1}} .
\end{gathered}
$$

[1] H. Ishimori, T. Kobayashi, H. Ohki, Y. Shimizu, H. Okada and M. Tanimoto, Prog. Theor. Phys. Suppl. 183, 1 (2010) doi:10.1143/PTPS.183.1 [arXiv:1003.3552 [hep-th]].

[2] G. Altarelli and F. Feruglio, Rev. Mod. Phys. 82, 2701 (2010) doi:10.1103/RevModPhys.82.2701 [arXiv:1002.0211 [hep$\mathrm{ph}]$.

[3] S. F. King and C. Luhn, Rept. Prog. Phys. 76, 056201 (2013) doi:10.1088/0034-4885/76/5/056201 [arXiv:1301.1340 [hep-ph]].

[4] S. F. King, A. Merle, S. Morisi, Y. Shimizu and M. Tanimoto, New J. Phys. 16, 045018 (2014) doi:10.1088/13672630/16/4/045018 [arXiv:1402.4271 [hep-ph]].

[5] S. F. King, Prog. Part. Nucl. Phys. 94, 217 (2017) doi:10.1016/j.ppnp.2017.01.003 [arXiv:1701.04413 [hep-ph]].

[6] J. M. Gerard, Z. Phys. C 18, 145 (1983). doi:10.1007/BF01572477

[7] J. Kubo, Phys. Lett. B 578, 156 (2004) Erratum: [Phys. Lett. B 619, 387 (2005)] doi:10.1016/j.physletb.2005.06.013, 10.1016/j.physletb.2003.10.048 [hep-ph/0309167].

[8] T. Kobayashi, J. Kubo and H. Terao, Phys. Lett. B 568, 83 (2003) doi:10.1016/j.physletb.2003.03.002 [hep-ph/0303084].

[9] S. L. Chen, M. Frigerio and E. Ma, Phys. Rev. D 70, 073008 (2004) Erratum: [Phys. Rev. D 70, 079905 (2004)] doi:10.1103/PhysRevD.70.079905, 10.1103/PhysRevD.70.073008 [hep-ph/0404084].

[10] A. Mondragon, M. Mondragon and E. Peinado, Phys. Rev. D 76, 076003 (2007) doi:10.1103/PhysRevD.76.076003 [arXiv:0706.0354 [hep-ph]].

[11] A. Mondragon, M. Mondragon and E. Peinado, Rev. Mex. Fis. 54, no. 3, 81 (2008) [Rev. Mex. Fis. Suppl. 54, 0181 (2008)] [arXiv:0805.3507 [hep-ph]].

[12] G. Bhattacharyya, P. Leser and H. Pas, Phys. Rev. D 83, 011701 (2011) doi:10.1103/PhysRevD.83.011701 [arXiv:1006.5597 [hep-ph]].

[13] P. V. Dong, H. N. Long, C. H. Nam and V. V. Vien, Phys. Rev. D 85, 053001 (2012) doi:10.1103/PhysRevD.85.053001 [arXiv:1111.6360 [hep-ph]].

[14] A. G. Dias, A. C. B. Machado and C. C. Nishi, Phys. Rev. D 86, 093005 (2012) doi:10.1103/PhysRevD.86.093005 [arXiv:1206.6362 [hep-ph]].

[15] D. Meloni, JHEP 1205, 124 (2012) doi:10.1007/JHEP05(2012)124 [arXiv:1203.3126 [hep-ph]].

[16] F. Gonzalez Canales, A. Mondragon and M. Mondragon, Fortsch. Phys. 61, 546 (2013) doi:10.1002/prop.201200121 [arXiv:1205.4755 [hep-ph]].

[17] F. González Canales, A. Mondragón, M. Mondragón, U. J. Saldaña Salazar and L. Velasco-Sevilla, Phys. Rev. D 88, 096004 (2013) doi:10.1103/PhysRevD.88.096004 [arXiv:1304.6644 [hep-ph]].

[18] E. Ma and B. Melic, Phys. Lett. B 725, 402 (2013) doi:10.1016/j.physletb.2013.07.015 [arXiv:1303.6928 [hep-ph]].

[19] Y. Kajiyama, H. Okada and K. Yagyu, Nucl. Phys. B 887, 358 (2014) doi:10.1016/j.nuclphysb.2014.08.009 [arXiv:1309.6234 [hep-ph]].

[20] A. E. Cárcamo Hernández, R. Martinez and F. Ochoa, Eur. Phys. J. C 76, no. 11, 634 (2016) doi:10.1140/epjc/s10052016-4480-3 [arXiv:1309.6567 [hep-ph]].

[21] E. Ma and R. Srivastava, Phys. Lett. B 741, 217 (2015) doi:10.1016/j.physletb.2014.12.049 [arXiv:1411.5042 [hep-ph]].

[22] A. E. Cárcamo Hernández, R. Martinez and J. Nisperuza, Eur. Phys. J. C 75, no. 2, 72 (2015) doi:10.1140/epjc/s10052015-3278-z [arXiv:1401.0937 [hep-ph]].

[23] A. E. Cárcamo Hernández, E. Cataño Mur and R. Martinez, Phys. Rev. D 90, no. 7 , 073001 (2014) doi:10.1103/PhysRevD.90.073001 [arXiv:1407.5217 [hep-ph]].

[24] S. Gupta, C. S. Kim and P. Sharma, Phys. Lett. B 740, 353 (2015) doi:10.1016/j.physletb.2014.12.005 [arXiv:1408.0172 [hep-ph]].

[25] A. E. Cárcamo Hernández, I. de Medeiros Varzielas and E. Schumacher, Phys. Rev. D 93, no. 1, 016003 (2016) doi:10.1103/PhysRevD.93.016003 [arXiv:1509.02083 [hep-ph]].

[26] A. E. Cárcamo Hernández, I. de Medeiros Varzielas and N. A. Neill, Phys. Rev. D 94, no. 3, 033011 (2016) doi:10.1103/PhysRevD.94.033011 [arXiv:1511.07420 [hep-ph]].

[27] A. E. Cárcamo Hernández, I. de Medeiros Varzielas and E. Schumacher, arXiv:1601.00661 [hep-ph].

[28] A. E. Cárcamo Hernández, Eur. Phys. J. C 76, no. 9, 503 (2016) doi:10.1140/epjc/s10052-016-4351-y [arXiv:1512.09092 [hep-ph]].

[29] A. E. Cárcamo Hernández, S. Kovalenko and I. Schmidt, JHEP 1702, 125 (2017) doi:10.1007/JHEP02(2017)125 [arXiv:1611.09797 [hep-ph]]. 
[30] C. Arbeláez, A. E. Cárcamo Hernández, S. Kovalenko and I. Schmidt, Eur. Phys. J. C 77, no. 6, 422 (2017) doi:10.1140/epjc/s10052-017-4948-9 [arXiv:1602.03607 [hep-ph]].

[31] J. C. Gómez-Izquierdo, Eur. Phys. J. C 77, no. 8, 551 (2017) doi:10.1140/epjc/s10052-017-5094-0 [arXiv:1701.01747 [hep-ph]].

[32] A. A. Cruz and M. Mondragón, arXiv:1701.07929 [hep-ph].

[33] E. Ma and G. Rajasekaran, Phys. Rev. D 64, 113012 (2001) doi:10.1103/PhysRevD.64.113012 [hep-ph/0106291].

[34] X. G. He, Y. Y. Keum and R. R. Volkas, JHEP 0604, 039 (2006) doi:10.1088/1126-6708/2006/04/039 [hep-ph/0601001].

[35] F. Feruglio, C. Hagedorn, Y. Lin and L. Merlo, Nucl. Phys. B 809, 218 (2009) doi:10.1016/j.nuclphysb.2008.10.002 [arXiv:0807.3160 [hep-ph]].

[36] F. Feruglio, C. Hagedorn, Y. Lin and L. Merlo, Nucl. Phys. B 832, 251 (2010) doi:10.1016/j.nuclphysb.2010.02.010 [arXiv:0911.3874 [hep-ph]].

[37] M. C. Chen and S. F. King, JHEP 0906, 072 (2009) doi:10.1088/1126-6708/2009/06/072 [arXiv:0903.0125 [hep-ph]].

[38] I. de Medeiros Varzielas and L. Merlo, JHEP 1102, 062 (2011) doi:10.1007/JHEP02(2011)062 [arXiv:1011.6662 [hep-ph]].

[39] G. Altarelli, F. Feruglio, L. Merlo and E. Stamou, JHEP 1208, 021 (2012) doi:10.1007/JHEP08(2012)021 [arXiv:1205.4670 [hep-ph]].

[40] Y. H. Ahn and S. K. Kang, Phys. Rev. D 86, 093003 (2012) doi:10.1103/PhysRevD.86.093003 [arXiv:1203.4185 [hep-ph]].

[41] N. Memenga, W. Rodejohann and H. Zhang, Phys. Rev. D 87, no. 5, 053021 (2013) doi:10.1103/PhysRevD.87.053021 [arXiv:1301.2963 [hep-ph]].

[42] R. Gonzalez Felipe, H. Serodio and J. P. Silva, Phys. Rev. D 88, no. 1, 015015 (2013) doi:10.1103/PhysRevD.88.015015 [arXiv:1304.3468 [hep-ph]].

[43] I. de Medeiros Varzielas and D. Pidt, JHEP 1303, 065 (2013) doi:10.1007/JHEP03(2013)065 [arXiv:1211.5370 [hep-ph]].

[44] H. Ishimori and E. Ma, Phys. Rev. D 86, 045030 (2012) doi:10.1103/PhysRevD.86.045030 [arXiv:1205.0075 [hep-ph]].

[45] S. F. King, S. Morisi, E. Peinado and J. W. F. Valle, Phys. Lett. B 724, 68 (2013) doi:10.1016/j.physletb.2013.05.067 [arXiv:1301.7065 [hep-ph]].

[46] A. E. Carcamo Hernandez, I. de Medeiros Varzielas, S. G. Kovalenko, H. Päs and I. Schmidt, Phys. Rev. D 88, no. 7, 076014 (2013) doi:10.1103/PhysRevD.88.076014 [arXiv:1307.6499 [hep-ph]].

[47] K. S. Babu, E. Ma and J. W. F. Valle, Phys. Lett. B 552, 207 (2003) doi:10.1016/S0370-2693(02)03153-2 [hep-ph/0206292].

[48] G. Altarelli and F. Feruglio, Nucl. Phys. B 741, 215 (2006) doi:10.1016/j.nuclphysb.2006.02.015 [hep-ph/0512103].

[49] S. Gupta, A. S. Joshipura and K. M. Patel, Phys. Rev. D 85, 031903 (2012) doi:10.1103/PhysRevD.85.031903 [arXiv:1112.6113 [hep-ph]].

[50] S. Morisi, M. Nebot, K. M. Patel, E. Peinado and J. W. F. Valle, Phys. Rev. D 88, 036001 (2013) doi:10.1103/PhysRevD.88.036001 [arXiv:1303.4394 [hep-ph]].

[51] G. Altarelli and F. Feruglio, Nucl. Phys. B 720, 64 (2005) doi:10.1016/j.nuclphysb.2005.05.005 [hep-ph/0504165].

[52] A. Kadosh and E. Pallante, JHEP 1008, 115 (2010) doi:10.1007/JHEP08(2010)115 [arXiv:1004.0321 [hep-ph]].

[53] A. Kadosh, JHEP 1306, 114 (2013) doi:10.1007/JHEP06(2013)114 [arXiv:1303.2645 [hep-ph]].

[54] F. del Aguila, A. Carmona and J. Santiago, JHEP 1008, 127 (2010) doi:10.1007/JHEP08(2010)127 [arXiv:1001.5151 [hep-ph]].

[55] M. D. Campos, A. E. Cárcamo Hernández, S. Kovalenko, I. Schmidt and E. Schumacher, Phys. Rev. D 90, no. 1, 016006 (2014) doi:10.1103/PhysRevD.90.016006 [arXiv:1403.2525 [hep-ph]].

[56] V. V. Vien and H. N. Long, Int. J. Mod. Phys. A 30, no. 21, 1550117 (2015) doi:10.1142/S0217751X15501171 [arXiv:1405.4665 [hep-ph]].

[57] A. S. Joshipura and K. M. Patel, Phys. Lett. B 749, 159 (2015) doi:10.1016/j.physletb.2015.07.062 [arXiv:1507.01235 [hep-ph]].

[58] A. E. Cárcamo Hernández and R. Martinez, Nucl. Phys. B 905, 337 (2016) doi:10.1016/j.nuclphysb.2016.02.025 [arXiv:1501.05937 [hep-ph]].

[59] B. Karmakar and A. Sil, Phys. Rev. D 96, no. 1, 015007 (2017) doi:10.1103/PhysRevD.96.015007 [arXiv:1610.01909 [hep-ph]].

[60] P. Chattopadhyay and K. M. Patel, Nucl. Phys. B 921, 487 (2017) doi:10.1016/j.nuclphysb.2017.06.008 [arXiv:1703.09541 [hep-ph]].

[61] A. E. Cárcamo Hernández and H. N. Long, J. Phys. G 45, no. 4, 045001 (2018) doi:10.1088/1361-6471/aaace7 [arXiv:1705.05246 [hep-ph]].

[62] E. Ma and G. Rajasekaran, EPL 119, no. 3, 31001 (2017) doi:10.1209/0295-5075/119/31001 [arXiv:1708.02208 [hep-ph]].

[63] S. Centelles Chuliá, R. Srivastava and J. W. F. Valle, Phys. Lett. B 773, 26 (2017) doi:10.1016/j.physletb.2017.07.065 [arXiv:1706.00210 [hep-ph]].

[64] F. Björkeroth, E. J. Chun and S. F. King, Phys. Lett. B 777, 428 (2018) doi:10.1016/j.physletb.2017.12.058 [arXiv:1711.05741 [hep-ph]].

[65] R. Srivastava, C. A. Ternes, M. Tórtola and J. W. F. Valle, Phys. Lett. B 778, 459 (2018) doi:10.1016/j.physletb.2018.01.014 [arXiv:1711.10318 [hep-ph]].

[66] D. Borah and B. Karmakar, Phys. Lett. B 780, 461 (2018) doi:10.1016/j.physletb.2018.03.047 [arXiv:1712.06407 [hep-ph]].

[67] A. S. Belyaev, S. F. King and P. B. Schaefers, Phys. Rev. D 97, no. 11, 115002 (2018) doi:10.1103/PhysRevD.97.115002 [arXiv:1801.00514 [hep-ph]].

[68] A. E. Cárcamo Hernández and S. F. King, Phys. Rev. D 99, no. 9, 095003 (2019) doi:10.1103/PhysRevD.99.095003 [arXiv:1803.07367 [hep-ph]].

[69] R. Srivastava, C. A. Ternes, M. Tórtola and J. W. F. Valle, Phys. Rev. D 97, no. 9, 095025 (2018) 
doi:10.1103/PhysRevD.97.095025 [arXiv:1803.10247 [hep-ph]].

[70] L. M. G. De La Vega, R. Ferro-Hernandez and E. Peinado, Phys. Rev. D 99, no. 5, 055044 (2019) doi:10.1103/PhysRevD.99.055044 [arXiv:1811.10619 [hep-ph]].

[71] D. Borah and B. Karmakar, Phys. Lett. B 789, 59 (2019) doi:10.1016/j.physletb.2018.12.006 [arXiv:1806.10685 [hep-ph]].

[72] S. Pramanick, arXiv:1903.04208 [hep-ph].

[73] A. E. Cárcamo Hernández, J. Marchant González and U. J. Saldaña-Salazar, arXiv:1904.09993 [hep-ph].

[74] A. E. Cárcamo Hernández, M. González and N. A. Neill, arXiv:1906.00978 [hep-ph].

[75] F. Bazzocchi, L. Merlo and S. Morisi, Phys. Rev. D 80, 053003 (2009) doi:10.1103/PhysRevD.80.053003 [arXiv:0902.2849 [hep-ph]].

[76] G. Altarelli, F. Feruglio and L. Merlo, JHEP 0905, 020 (2009) doi:10.1088/1126-6708/2009/05/020 [arXiv:0903.1940 [hep-ph]].

[77] R. de Adelhart Toorop, F. Bazzocchi and L. Merlo, JHEP 1008, 001 (2010) doi:10.1007/JHEP08(2010)001 [arXiv:1003.4502 [hep-ph]].

[78] K. M. Patel, Phys. Lett. B 695, 225 (2011) doi:10.1016/j.physletb.2010.11.024 [arXiv:1008.5061 [hep-ph]].

[79] S. Morisi, K. M. Patel and E. Peinado, Phys. Rev. D 84, 053002 (2011) doi:10.1103/PhysRevD.84.053002 [arXiv:1107.0696 [hep-ph]].

[80] R. N. Mohapatra and C. C. Nishi, Phys. Rev. D 86, 073007 (2012) doi:10.1103/PhysRevD.86.073007 [arXiv:1208.2875 [hep-ph]].

[81] P. S. Bhupal Dev, B. Dutta, R. N. Mohapatra and M. Severson, Phys. Rev. D 86, 035002 (2012) doi:10.1103/PhysRevD.86.035002 [arXiv:1202.4012 [hep-ph]].

[82] I. de Medeiros Varzielas and L. Lavoura, J. Phys. G 40, 085002 (2013) doi:10.1088/0954-3899/40/8/085002 [arXiv:1212.3247 [hep-ph]].

[83] G. J. Ding, S. F. King, C. Luhn and A. J. Stuart, JHEP 1305, 084 (2013) doi:10.1007/JHEP05(2013)084 [arXiv:1303.6180 [hep-ph]].

[84] H. Ishimori, Y. Shimizu, M. Tanimoto and A. Watanabe, Phys. Rev. D 83, 033004 (2011) doi:10.1103/PhysRevD.83.033004 [arXiv:1010.3805 [hep-ph]].

[85] G. J. Ding and Y. L. Zhou, Nucl. Phys. B 876, 418 (2013) doi:10.1016/j.nuclphysb.2013.08.011 [arXiv:1304.2645 [hep-ph]].

[86] C. Hagedorn and M. Serone, JHEP 1110, 083 (2011) doi:10.1007/JHEP10(2011)083 [arXiv:1106.4021 [hep-ph]].

[87] M. D. Campos, A. E. Cárcamo Hernández, H. Päs and E. Schumacher, Phys. Rev. D 91, no. 11, 116011 (2015) doi:10.1103/PhysRevD.91.116011 [arXiv:1408.1652 [hep-ph]].

[88] P. V. Dong, H. N. Long, D. V. Soa and V. V. Vien, Eur. Phys. J. C 71, 1544 (2011) doi:10.1140/epjc/s10052-011-1544-2 [arXiv:1009.2328 [hep-ph]].

[89] V. V. Vien, H. N. Long and D. P. Khoi, Int. J. Mod. Phys. A 30, no. 17, 1550102 (2015) doi:10.1142/S0217751X1550102X [arXiv:1506.06063 [hep-ph]].

[90] F. J. de Anda, S. F. King and E. Perdomo, JHEP 1712, 075 (2017) Erratum: [JHEP 1904, 069 (2019)] doi:10.1007/JHEP12(2017)075, 10.1007/JHEP04(2019)069 [arXiv:1710.03229 [hep-ph]].

[91] F. J. de Anda and S. F. King, JHEP 1807, 057 (2018) doi:10.1007/JHEP07(2018)057 [arXiv:1803.04978 [hep-ph]].

[92] A. E. Cárcamo Hernández and S. F. King, arXiv:1903.02565 [hep-ph].

[93] A. E. Cárcamo Hernández, N. A. Pérez-Julve and Y. Hidalgo Velásquez, arXiv:1907.13083 [hep-ph].

[94] P. H. Frampton and T. W. Kephart, Int. J. Mod. Phys. A 10, 4689 (1995) doi:10.1142/S0217751X95002187 [hep$\mathrm{ph} / 9409330]$.

[95] W. Grimus and L. Lavoura, Phys. Lett. B 572, 189 (2003) doi:10.1016/j.physletb.2003.08.032 [hep-ph/0305046].

[96] W. Grimus, A. S. Joshipura, S. Kaneko, L. Lavoura and M. Tanimoto, JHEP 0407, 078 (2004) doi:10.1088/11266708/2004/07/078 [hep-ph/0407112].

[97] M. Frigerio, S. Kaneko, E. Ma and M. Tanimoto, Phys. Rev. D 71, 011901 (2005) doi:10.1103/PhysRevD.71.011901 [hep-ph/0409187].

[98] A. Adulpravitchai, A. Blum and C. Hagedorn, JHEP 0903, 046 (2009) doi:10.1088/1126-6708/2009/03/046 [arXiv:0812.3799 [hep-ph]].

[99] H. Ishimori, T. Kobayashi, H. Ohki, Y. Omura, R. Takahashi and M. Tanimoto, Phys. Lett. B 662, 178 (2008) doi:10.1016/j.physletb.2008.03.007 [arXiv:0802.2310 [hep-ph]].

[100] C. Hagedorn and R. Ziegler, Phys. Rev. D 82, 053011 (2010) doi:10.1103/PhysRevD.82.053011 [arXiv:1007.1888 [hep-ph]].

[101] D. Meloni, S. Morisi and E. Peinado, Phys. Lett. B 703, 281 (2011) doi:10.1016/j.physletb.2011.07.084 [arXiv:1104.0178 [hep-ph]].

[102] V. V. Vien and H. N. Long, Int. J. Mod. Phys. A 28, 1350159 (2013) doi:10.1142/S0217751X13501595 [arXiv:1312.5034 [hep-ph]].

[103] K. S. Babu and J. Kubo, Phys. Rev. D 71, 056006 (2005) doi:10.1103/PhysRevD.71.056006 [hep-ph/0411226].

[104] Y. Kajiyama, E. Itou and J. Kubo, Nucl. Phys. B 743, 74 (2006) doi:10.1016/j.nuclphysb.2006.02.042 [hep-ph/0511268].

[105] Y. Kajiyama, JHEP 0704, 007 (2007) doi:10.1088/1126-6708/2007/04/007 [hep-ph/0702056 [HEP-PH]].

[106] N. Kifune, J. Kubo and A. Lenz, Phys. Rev. D 77, 076010 (2008) doi:10.1103/PhysRevD.77.076010 [arXiv:0712.0503 [hep-ph]].

[107] K. S. Babu and Y. Meng, Phys. Rev. D 80, 075003 (2009) doi:10.1103/PhysRevD.80.075003 [arXiv:0907.4231 [hep-ph]].

[108] K. Kawashima, J. Kubo and A. Lenz, Phys. Lett. B 681, 60 (2009) doi:10.1016/j.physletb.2009.09.064 [arXiv:0907.2302 [hep-ph]].

[109] Y. Kaburaki, K. Konya, J. Kubo and A. Lenz, Phys. Rev. D 84, 016007 (2011) doi:10.1103/PhysRevD.84.016007 
[arXiv:1012.2435 [hep-ph]].

[110] K. S. Babu, K. Kawashima and J. Kubo, Phys. Rev. D 83, 095008 (2011) doi:10.1103/PhysRevD.83.095008 [arXiv:1103.1664 [hep-ph]].

[111] T. Araki and Y. F. Li, Phys. Rev. D 85, 065016 (2012) doi:10.1103/PhysRevD.85.065016 [arXiv:1112.5819 [hep-ph]].

[112] J. C. Gómez-Izquierdo, F. González-Canales and M. Mondragon, Eur. Phys. J. C 75, no. 5, 221 (2015) doi:10.1140/epjc/s10052-015-3440-7 [arXiv:1312.7385 [hep-ph]].

[113] J. C. Gómez-Izquierdo, F. Gonzalez-Canales and M. Mondragón, Int. J. Mod. Phys. A 32, no. 28-29, 1750171 (2017) doi:10.1142/S0217751X17501718 [arXiv:1705.06324 [hep-ph]].

[114] C. Luhn, S. Nasri and P. Ramond, Phys. Lett. B 652, 27 (2007) doi:10.1016/j.physletb.2007.06.059 [arXiv:0706.2341 [hep-ph]].

[115] C. Hagedorn, M. A. Schmidt and A. Y. Smirnov, Phys. Rev. D 79, 036002 (2009) doi:10.1103/PhysRevD.79.036002 [arXiv:0811.2955 [hep-ph]].

[116] Q. H. Cao, S. Khalil, E. Ma and H. Okada, Phys. Rev. Lett. 106, 131801 (2011) doi:10.1103/PhysRevLett.106.131801 [arXiv:1009.5415 [hep-ph]].

[117] C. Luhn, K. M. Parattu and A. Wingerter, JHEP 1212, 096 (2012) doi:10.1007/JHEP12(2012)096 [arXiv:1210.1197 [hep-ph]].

[118] Y. Kajiyama, H. Okada and K. Yagyu, JHEP 1310, 196 (2013) doi:10.1007/JHEP10(2013)196 [arXiv:1307.0480 [hep-ph]].

[119] C. Bonilla, S. Morisi, E. Peinado and J. W. F. Valle, Phys. Lett. B 742, 99 (2015) doi:10.1016/j.physletb.2015.01.017 [arXiv:1411.4883 [hep-ph]].

[120] V. V. Vien and H. N. Long, JHEP 1404, 133 (2014) doi:10.1007/JHEP04(2014)133 [arXiv:1402.1256 [hep-ph]].

[121] V. V. Vien, Mod. Phys. Lett. A 29, 28 (2014) doi:10.1142/S0217732314501399 [arXiv:1508.02585 [hep-ph]].

[122] A. E. Cárcamo Hernández and R. Martinez, J. Phys. G 43, no. 4, 045003 (2016) doi:10.1088/0954-3899/43/4/045003 [arXiv:1501.07261 [hep-ph]].

[123] C. Arbeláez, A. E. Cárcamo Hernández, S. Kovalenko and I. Schmidt, Phys. Rev. D 92, no. 11, 115015 (2015) doi:10.1103/PhysRevD.92.115015 [arXiv:1507.03852 [hep-ph]].

[124] G. J. Ding, Nucl. Phys. B 853, 635 (2011) doi:10.1016/j.nuclphysb.2011.08.012 [arXiv:1105.5879 [hep-ph]].

[125] C. Hartmann, Phys. Rev. D 85, 013012 (2012) doi:10.1103/PhysRevD.85.013012 [arXiv:1109.5143 [hep-ph]].

[126] C. Hartmann and A. Zee, Nucl. Phys. B 853, 105 (2011) doi:10.1016/j.nuclphysb.2011.07.023 [arXiv:1106.0333 [hep-ph]].

[127] Y. Kajiyama and H. Okada, Nucl. Phys. B 848, 303 (2011) doi:10.1016/j.nuclphysb.2011.02.020 [arXiv:1011.5753 [hep$\mathrm{ph}]$.

[128] A. Aranda, C. D. Carone and R. F. Lebed, Phys. Rev. D 62, 016009 (2000) doi:10.1103/PhysRevD.62.016009 [hep$\mathrm{ph} / 0002044]$.

[129] F. Feruglio, C. Hagedorn, Y. Lin and L. Merlo, Nucl. Phys. B 775, 120 (2007) Erratum: [Nucl. Phys. B 836, 127 (2010)] doi:10.1016/j.nuclphysb.2007.04.002, 10.1016/j.nuclphysb.2010.04.018 [hep-ph/0702194].

[130] S. Sen, Phys. Rev. D 76, 115020 (2007) doi:10.1103/PhysRevD.76.115020 [arXiv:0710.2734 [hep-ph]].

[131] A. Aranda, Phys. Rev. D 76, 111301 (2007) doi:10.1103/PhysRevD.76.111301 [arXiv:0707.3661 [hep-ph]].

[132] M. C. Chen and K. T. Mahanthappa, Phys. Lett. B 652, 34 (2007) doi:10.1016/j.physletb.2007.06.064 [arXiv:0705.0714 [hep-ph]].

[133] D. A. Eby, P. H. Frampton and S. Matsuzaki, Phys. Lett. B 671, 386 (2009) doi:10.1016/j.physletb.2008.11.074 [arXiv:0810.4899 [hep-ph]].

[134] P. H. Frampton, T. W. Kephart and S. Matsuzaki, Phys. Rev. D 78, 073004 (2008) doi:10.1103/PhysRevD.78.073004 [arXiv:0807.4713 [hep-ph]].

[135] P. H. Frampton and S. Matsuzaki, Mod. Phys. Lett. A 24, 429 (2009) doi:10.1142/S0217732309030229 [arXiv:0807.4785 [hep-ph]].

[136] D. A. Eby, P. H. Frampton and S. Matsuzaki, Phys. Rev. D 80, 053007 (2009) doi:10.1103/PhysRevD.80.053007 [arXiv:0907.3425 [hep-ph]].

[137] P. H. Frampton and S. Matsuzaki, Phys. Lett. B 679, 347 (2009) doi:10.1016/j.physletb.2009.08.001 [arXiv:0902.1140 [hep-ph]].

[138] L. Merlo, S. Rigolin and B. Zaldivar, JHEP 1111, 047 (2011) doi:10.1007/JHEP11(2011)047 [arXiv:1108.1795 [hep-ph]].

[139] D. A. Eby, P. H. Frampton, X. G. He and T. W. Kephart, Phys. Rev. D 84, 037302 (2011) doi:10.1103/PhysRevD.84.037302 [arXiv:1103.5737 [hep-ph]].

[140] D. A. Eby and P. H. Frampton, Phys. Lett. B 713, 249 (2012) doi:10.1016/j.physletb.2012.06.004 [arXiv:1111.4938 [hep$\mathrm{ph}]$.

[141] M. C. Chen and K. T. Mahanthappa, arXiv:1107.3856 [hep-ph].

[142] A. Meroni, S. T. Petcov and M. Spinrath, Phys. Rev. D 86, 113003 (2012) doi:10.1103/PhysRevD.86.113003 [arXiv:1205.5241 [hep-ph]].

[143] P. H. Frampton, C. M. Ho and T. W. Kephart, Phys. Rev. D 89, no. 2, 027701 (2014) doi:10.1103/PhysRevD.89.027701 [arXiv:1305.4402 [hep-ph]].

[144] M. C. Chen, J. Huang, K. T. Mahanthappa and A. M. Wijangco, JHEP 1310, 112 (2013) doi:10.1007/JHEP10(2013)112 [arXiv:1307.7711 [hep-ph]].

[145] I. Girardi, A. Meroni, S. T. Petcov and M. Spinrath, JHEP 1402, 050 (2014) doi:10.1007/JHEP02(2014)050 [arXiv:1312.1966 [hep-ph]].

[146] C. D. Carone, S. Chaurasia and S. Vasquez, Phys. Rev. D 95, no. 1, 015025 (2017) doi:10.1103/PhysRevD.95.015025 [arXiv:1611.00784 [hep-ph]]. 
[147] V. V. Vien, H. N. Long and A. E. Cárcamo Hernández, Mod. Phys. Lett. A 34, no. 01, 1950005 (2019) doi:10.1142/S0217732319500056 [arXiv:1812.07263 [hep-ph]].

[148] C. D. Carone and M. Merchand, arXiv:1904.11059 [hep-ph].

[149] A. E. Cárcamo Hernández, Y. Hidalgo Velásquez and N. A. Pérez-Julve, arXiv:1905.02323 [hep-ph].

[150] G. C. Branco, J. M. Gerard and W. Grimus, Phys. Lett. 136B, 383 (1984). doi:10.1016/0370-2693(84)92024-0

[151] I. de Medeiros Varzielas, S. F. King and G. G. Ross, Phys. Lett. B 648, 201 (2007) doi:10.1016/j.physletb.2007.03.009 [hep-ph/0607045].

[152] E. Ma, Phys. Lett. B 660, 505 (2008) doi:10.1016/j.physletb.2007.12.060 [arXiv:0709.0507 [hep-ph]].

[153] I. de Medeiros Varzielas, D. Emmanuel-Costa and P. Leser, Phys. Lett. B 716, 193 (2012) doi:10.1016/j.physletb.2012.08.008 [arXiv:1204.3633 [hep-ph]].

[154] G. Bhattacharyya, I. de Medeiros Varzielas and P. Leser, Phys. Rev. Lett. 109, 241603 (2012) doi:10.1103/PhysRevLett.109.241603 [arXiv:1210.0545 [hep-ph]].

[155] P. M. Ferreira, W. Grimus, L. Lavoura and P. O. Ludl, JHEP 1209, 128 (2012) doi:10.1007/JHEP09(2012)128 [arXiv:1206.7072 [hep-ph]].

[156] E. Ma, Phys. Lett. B 723, 161 (2013) doi:10.1016/j.physletb.2013.05.011 [arXiv:1304.1603 [hep-ph]].

[157] C. C. Nishi, Phys. Rev. D 88, no. 3, 033010 (2013) doi:10.1103/PhysRevD.88.033010 [arXiv:1306.0877 [hep-ph]].

[158] I. de Medeiros Varzielas and D. Pidt, J. Phys. G 41, 025004 (2014) doi:10.1088/0954-3899/41/2/025004 [arXiv:1307.0711 [hep-ph]].

[159] A. Aranda, C. Bonilla, S. Morisi, E. Peinado and J. W. F. Valle, Phys. Rev. D 89, no. 3, 033001 (2014) doi:10.1103/PhysRevD.89.033001 [arXiv:1307.3553 [hep-ph]].

[160] P. F. Harrison, R. Krishnan and W. G. Scott, Int. J. Mod. Phys. A 29, no. 18, 1450095 (2014) doi:10.1142/S0217751X1450095X [arXiv:1406.2025 [hep-ph]].

[161] E. Ma and A. Natale, Phys. Lett. B 734, 403 (2014) doi:10.1016/j.physletb.2014.05.070 [arXiv:1403.6772 [hep-ph]].

[162] M. Abbas and S. Khalil, Phys. Rev. D 91, no. 5, 053003 (2015) doi:10.1103/PhysRevD.91.053003 [arXiv:1406.6716 [hep$\mathrm{ph}]$.

[163] M. Abbas, S. Khalil, A. Rashed and A. Sil, Phys. Rev. D 93, no. 1, 013018 (2016) doi:10.1103/PhysRevD.93.013018 [arXiv:1508.03727 [hep-ph]].

[164] I. de Medeiros Varzielas, JHEP 1508, 157 (2015) doi:10.1007/JHEP08(2015)157 [arXiv:1507.00338 [hep-ph]].

[165] F. Björkeroth, F. J. de Anda, I. de Medeiros Varzielas and S. F. King, Phys. Rev. D 94, no. 1, 016006 (2016) doi:10.1103/PhysRevD.94.016006 [arXiv:1512.00850 [hep-ph]].

[166] P. Chen, G. J. Ding, A. D. Rojas, C. A. Vaquera-Araujo and J. W. F. Valle, JHEP 1601, 007 (2016) doi:10.1007/JHEP01(2016)007 [arXiv:1509.06683 [hep-ph]].

[167] V. V. Vien, A. E. Cárcamo Hernández and H. N. Long, Nucl. Phys. B 913, 792 (2016) doi:10.1016/j.nuclphysb.2016.10.010 [arXiv:1601.03300 [hep-ph]].

[168] A. E. Cárcamo Hernández, H. N. Long and V. V. Vien, Eur. Phys. J. C 76, no. 5, 242 (2016) doi:10.1140/epjc/s10052016-4074-0 [arXiv:1601.05062 [hep-ph]].

[169] A. E. Cárcamo Hernández, S. Kovalenko, J. W. F. Valle and C. A. Vaquera-Araujo, JHEP 1707, 118 (2017) doi:10.1007/JHEP07(2017)118 [arXiv:1705.06320 [hep-ph]].

[170] I. de Medeiros Varzielas, G. G. Ross and J. Talbert, JHEP 1803, 007 (2018) doi:10.1007/JHEP03(2018)007 [arXiv:1710.01741 [hep-ph]].

[171] N. Bernal, A. E. Cárcamo Hernández, I. de Medeiros Varzielas and S. Kovalenko, JHEP 1805, 053 (2018) doi:10.1007/JHEP05(2018)053 [arXiv:1712.02792 [hep-ph]].

[172] A. E. Cárcamo Hernández, H. N. Long and V. V. Vien, Eur. Phys. J. C 78, no. 10, 804 (2018) doi:10.1140/epjc/s10052018-6284-0 [arXiv:1803.01636 [hep-ph]].

[173] I. De Medeiros Varzielas, M. L. López-Ibáñez, A. Melis and O. Vives, JHEP 1809, 047 (2018) doi:10.1007/JHEP09(2018)047 [arXiv:1807.00860 [hep-ph]].

[174] A. E. Cárcamo Hernández, S. Kovalenko, J. W. F. Valle and C. A. Vaquera-Araujo, JHEP 1902, 065 (2019) doi:10.1007/JHEP02(2019)065 [arXiv:1811.03018 [hep-ph]].

[175] A. E. Cárcamo Hernández, J. C. Gómez-Izquierdo, S. Kovalenko and M. Mondragón, Nucl. Phys. B 946, 114688 (2019) doi:10.1016/j.nuclphysb.2019.114688 [arXiv:1810.01764 [hep-ph]].

[176] F. Björkeroth, I. de Medeiros Varzielas, M. L. López-Ibáñez, A. Melis and Ó. Vives, arXiv:1904.10545 [hep-ph].

[177] S. F. King, C. Luhn and A. J. Stuart, Nucl. Phys. B 867, 203 (2013) doi:10.1016/j.nuclphysb.2012.09.021 [arXiv:1207.5741 [hep-ph]].

[178] S. F. King, T. Neder and A. J. Stuart, Phys. Lett. B 726, 312 (2013) doi:10.1016/j.physletb.2013.08.052 [arXiv:1305.3200 [hep-ph]].

[179] G. J. Ding and S. F. King, Phys. Rev. D 89, no. 9, 093020 (2014) doi:10.1103/PhysRevD.89.093020 [arXiv:1403.5846 [hep-ph]].

[180] H. Ishimori and S. F. King, Phys. Lett. B 735, 33 (2014) doi:10.1016/j.physletb.2014.06.003 [arXiv:1403.4395 [hep-ph]].

[181] S. F. King and T. Neder, Phys. Lett. B 736, 308 (2014) doi:10.1016/j.physletb.2014.07.043 [arXiv:1403.1758 [hep-ph]].

[182] H. Ishimori, S. F. King, H. Okada and M. Tanimoto, Phys. Lett. B 743, 172 (2015) doi:10.1016/j.physletb.2015.02.027 [arXiv:1411.5845 [hep-ph]].

[183] L. L. Everett and A. J. Stuart, Phys. Rev. D 79, 085005 (2009) doi:10.1103/PhysRevD.79.085005 [arXiv:0812.1057 [hep$\mathrm{ph}]$. 
[184] F. Feruglio and A. Paris, JHEP 1103, 101 (2011) doi:10.1007/JHEP03(2011)101 [arXiv:1101.0393 [hep-ph]].

[185] I. K. Cooper, S. F. King and A. J. Stuart, Nucl. Phys. B 875, 650 (2013) doi:10.1016/j.nuclphysb.2013.07.027 [arXiv:1212.1066 [hep-ph]].

[186] I. de Medeiros Varzielas and L. Lavoura, J. Phys. G 41, 055005 (2014) doi:10.1088/0954-3899/41/5/055005 [arXiv:1312.0215 [hep-ph]].

[187] J. Gehrlein, J. P. Oppermann, D. Schäfer and M. Spinrath, Nucl. Phys. B 890, 539 (2014) doi:10.1016/j.nuclphysb.2014.11.023 [arXiv:1410.2057 [hep-ph]].

[188] J. Gehrlein, S. T. Petcov, M. Spinrath and X. Zhang, Nucl. Phys. B 896, 311 (2015) doi:10.1016/j.nuclphysb.2015.04.019 [arXiv:1502.00110 [hep-ph]].

[189] A. Di Iura, C. Hagedorn and D. Meloni, JHEP 1508, 037 (2015) doi:10.1007/JHEP08(2015)037 [arXiv:1503.04140 [hep$\mathrm{ph}]$.

[190] P. Ballett, S. Pascoli and J. Turner, Phys. Rev. D 92, no. 9, 093008 (2015) doi:10.1103/PhysRevD.92.093008 [arXiv:1503.07543 [hep-ph]].

[191] J. Gehrlein, S. T. Petcov, M. Spinrath and X. Zhang, Nucl. Phys. B 899, 617 (2015) doi:10.1016/j.nuclphysb.2015.08.019 [arXiv:1508.07930 [hep-ph]].

[192] J. Turner, Phys. Rev. D 92, no. 11, 116007 (2015) doi:10.1103/PhysRevD.92.116007 [arXiv:1507.06224 [hep-ph]].

[193] C. C. Li and G. J. Ding, JHEP 1505, 100 (2015) doi:10.1007/JHEP05(2015)100 [arXiv:1503.03711 [hep-ph]].

[194] G. J. Ding, S. F. King and C. C. Li, Nucl. Phys. B 925, 470 (2017) doi:10.1016/j.nuclphysb.2017.10.019 [arXiv:1705.05307 $[$ hep-ph]].

[195] M. V. Chizhov and G. Dvali, Phys. Lett. B 703, 593 (2011) doi:10.1016/j.physletb.2011.08.056 [arXiv:0908.0924 [hep-ph]].

[196] N. Maru, N. Okada and S. Okada, Phys. Rev. D 98, no. 7, 075021 (2018) doi:10.1103/PhysRevD.98.075021 [arXiv:1803.01274 [hep-ph]]

[197] K. Agashe, A. Azatov, T. Han, Y. Li, Z. G. Si and L. Zhu, Phys. Rev. D 81, 096002 (2010) doi:10.1103/PhysRevD.81.096002 [arXiv:0911.0059 [hep-ph]].

[198] D. Buarque Franzosi, G. Cacciapaglia, H. Cai, A. Deandrea and M. Frandsen, JHEP 1611, 076 (2016) doi:10.1007/JHEP11(2016)076 [arXiv:1605.01363 [hep-ph]].

[199] B. D. Sáez, F. Rojas-Abatte and A. R. Zerwekh, Phys. Rev. D 99, no. 7, 075026 (2019) doi:10.1103/PhysRevD.99.075026 [arXiv:1810.06375 [hep-ph]].

[200] K. Bora, Horizon 2, 112 (2013) [arXiv:1206.5909 [hep-ph]].

[201] Z. z. Xing, H. Zhang and S. Zhou, Phys. Rev. D 77, 113016 (2008) doi:10.1103/PhysRevD.77.113016 [arXiv:0712.1419 [hep-ph]].

[202] C. Patrignani et al. [Particle Data Group], Chin. Phys. C 40, no. 10, 100001 (2016). doi:10.1088/1674-1137/40/10/100001

[203] P. F. de Salas, D. V. Forero, C. A. Ternes, M. Tortola and J. W. F. Valle, Phys. Lett. B 782, 633 (2018) doi:10.1016/j.physletb.2018.06.019 [arXiv:1708.01186 [hep-ph]].

[204] A. R. Zerwekh, Eur. Phys. J. C 46, 791 (2006) doi:10.1140/epjc/s2006-02518-6 [hep-ph/0512261].

[205] R. Barbieri, A. E. Carcamo Hernandez, G. Corcella, R. Torre and E. Trincherini, JHEP 1003, 068 (2010) doi:10.1007/JHEP03(2010)068 [arXiv:0911.1942 [hep-ph]].

[206] A. R. Zerwekh, Mod. Phys. Lett. A 25, 423 (2010) doi:10.1142/S0217732310032627 [arXiv:0907.4690 [hep-ph]].

[207] A. E. Carcamo Hernandez and R. Torre, Nucl. Phys. B 841, 188 (2010) doi:10.1016/j.nuclphysb.2010.08.004 [arXiv:1005.3809 [hep-ph]].

[208] A. E. Carcamo Hernandez, Eur. Phys. J. C 72, 2154 (2012) doi:10.1140/epjc/s10052-012-2154-3 [arXiv:1008.1039 [hep$\mathrm{ph}]$.

[209] O. Castillo-Felisola, C. Corral, M. González, G. Moreno, N. A. Neill, F. Rojas, J. Zamora and A. R. Zerwekh, Eur. Phys. J. C 73, no. 12, 2669 (2013) doi:10.1140/epjc/s10052-013-2669-2 [arXiv:1308.1825 [hep-ph]].

[210] A. E. Cárcamo Hernández, B. Díaz Sáez, C. O. Dib and A. Zerwekh, Phys. Rev. D 96, no. 11, 115027 (2017) doi:10.1103/PhysRevD.96.115027 [arXiv:1707.05195 [hep-ph]].

[211] J. Urbina and A. R. Zerwekh, Nucl. Phys. B 934, 653 (2018) doi:10.1016/j.nuclphysb.2018.07.014 [arXiv:1803.08116 [hep-ph]].

[212] A. Das, N. Okada and D. Raut, Phys. Rev. D 97, no. 11, 115023 (2018) doi:10.1103/PhysRevD.97.115023 [arXiv:1710.03377 [hep-ph]].

[213] A. Das, N. Okada and D. Raut, Eur. Phys. J. C 78, no. 9, 696 (2018) doi:10.1140/epjc/s10052-018-6171-8 [arXiv:1711.09896 [hep-ph]].

[214] A. Das, T. Nomura, H. Okada and S. Roy, Phys. Rev. D 96, no. 7, 075001 (2017) doi:10.1103/PhysRevD.96.075001 [arXiv:1704.02078 [hep-ph]].

[215] F. Alessandria et al. [CUORE Collaboration], arXiv:1109.0494 [nucl-ex].

[216] A. Gando et al. [KamLAND-Zen Collaboration], Phys. Rev. Lett. 117, no. 8, 082503 (2016) Addendum: [Phys. Rev. Lett. 117, no. 10, 109903 (2016)] doi:10.1103/PhysRevLett.117.109903, 10.1103/PhysRevLett.117.082503 [arXiv:1605.02889 [hep-ex]].

[217] A. Ilakovac and A. Pilaftsis, Nucl. Phys. B 437, 491 (1995) doi:10.1016/0550-3213(94)00567-X [hep-ph/9403398].

[218] F. Deppisch and J. W. F. Valle, Phys. Rev. D 72, 036001 (2005) doi:10.1103/PhysRevD.72.036001 [hep-ph/0406040].

[219] M. Lindner, M. Platscher and F. S. Queiroz, Phys. Rept. 731, 1 (2018) doi:10.1016/j.physrep.2017.12.001 [arXiv:1610.06587 [hep-ph]]. 